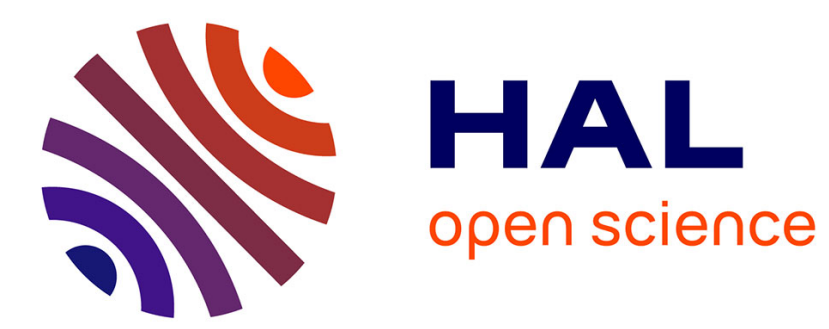

\title{
Motivic double shuffle
}

Ismaël Soudères

\section{To cite this version:}

Ismaël Soudères. Motivic double shuffle. International Journal of Number Theory, 2010, 6 (2), pp.339370. 10.1142/S1793042110002995 . hal-00308493v3

\section{HAL Id: hal-00308493 https://hal.science/hal-00308493v3}

Submitted on 17 Nov 2008

HAL is a multi-disciplinary open access archive for the deposit and dissemination of scientific research documents, whether they are published or not. The documents may come from teaching and research institutions in France or abroad, or from public or private research centers.
L'archive ouverte pluridisciplinaire HAL, est destinée au dépôt et à la diffusion de documents scientifiques de niveau recherche, publiés ou non, émanant des établissements d'enseignement et de recherche français ou étrangers, des laboratoires publics ou privés. 


\title{
MOTIVIC DOUBLE SHUFFLE
}

\author{
ISMAEL SOUDĖRES
}

\section{Contents}

Introduction 1

1. Integral representation of the double shuffle relations 2

1.1. Series representation of the stuffle relations 2

1.2. Integral representation of the shuffle relations 2

1.3. The stuffle relations in terms of integrals 3

2. Moduli spaces of curves; double shuffle and forgetful maps 6

$\begin{array}{ll}2.1 \text {. Shuffle and moduli spaces of curves } & 6\end{array}$

2.2. Stuffle and moduli spaces of curves 7

3. Motivic shuffle for the "convergent" words 8

3.1. Framed mixed Tate motives and motivic multiple zeta values 8

3.2. Motivic Shuffle 10

4. The stuffle case 12

4.1. Blow-up preliminaries $\quad 12$

4.2. The space $X_{n}$ and some of its properties 15

4.3. An alternative definition for motivic MZV 21

4.4. Motivic Stuffle 23

$\begin{array}{ll}\text { References } & 26\end{array}$

\section{INTRODUCTION}

For a $p$-tuple $\mathbf{k}=\left(k_{1}, \ldots, k_{p}\right)$ of positive integers and $k_{1} \geqslant 2$, the multiple zeta value $\zeta(\mathbf{k})$ is defined as

$$
\zeta(\mathbf{k})=\sum_{n_{1}>\ldots>n_{p}>0} \frac{1}{n_{1}^{k_{1}} \cdots n_{p}^{k_{p}}} .
$$

These values satisfy two families of algebraic (quadratic) relations known as double shuffle relations, or shuffle and stuffle, described below.

In [GM04] A.B. Goncharov and Y. Manin define a motivic version of multiple zeta values using certain framed mixed Tate motives attached to moduli spaces of genus 0 curves. In this context, the real multiple zeta values appear naturally as periods of those motives attached to the moduli spaces of curves. They do not prove the double shuffle relations directly, referring instead to previous work by A.B. Goncharov in which, using a different definition of motivic multiple polylogarithms based on $\left(\mathbb{P}^{1}\right)^{n}$ rather than moduli spaces, the motivic double shuffle relations are shown via results on variations of mixed Hodge structure.

The goal of this article is to give an elementary proof of the double shuffle relations directly for the Goncharov and Manin motivic multiple zeta values. The

Date: November 17, 2008.

this work has been partially supported by a Marie Curie Early Stage Training fellowship for the AAG network at Durham University. 
shuffle relation (Proposition 3.8) is straightforward, but for the stuffle (Proposition 4.24) we use a modification of a method first introduced by $\mathrm{P}$. Cartier for the purpose of proving stuffle for the real multiple zeta values via integrals and blowup sequences. In this article, we will work over the base field $\mathbb{Q}$.

\section{Integral REPRESEntation of the Double Shuffle RELATions}

1.1. Series representation of the stuffle relations. The stuffle product of a $p$-tuple $\mathbf{k}=\left(k_{1}, \ldots, k_{p}\right)$ and a $q$-tuple $\mathbf{l}=\left(l_{1}, \ldots, l_{q}\right)$ is defined recursively by the formula:

$$
\begin{aligned}
\mathbf{k} * \mathbf{l}=\left(\mathbf{k} *\left(l_{1}, \ldots, l_{q-1}\right)\right) \cdot l_{q}+ & \left(\left(k_{1}, \ldots, k_{p-1}\right) * \mathbf{l}\right) \cdot k_{p} \\
+ & \left(\left(k_{1}, \ldots, k_{p-1}\right) *\left(l_{1}, \ldots, l_{q-1}\right)\right) \cdot\left(k_{p}+l_{q}\right)
\end{aligned}
$$

and $\mathbf{k} *()=() * \mathbf{k}=\mathbf{k}$. Here the + is a formal sum, $A \cdot a$ means that we concatenate $a$ at the end of the tuple $A$ and $\cdot$ is linear in $A$.

Let $\mathbf{k}$ and $\mathbf{l}$ be two such tuples of integers. We will write $\operatorname{st}(\mathbf{k}, \mathbf{l})$ for the set of individual terms in the formal sum $\mathbf{k} * \mathbf{l}$ whose coefficients are all equal to 1 . Such a generic term is then denoted by $\sigma \in \operatorname{st}(\mathbf{k}, \mathbf{l})$.

In order to multiply two multiple zeta values $\zeta(\mathbf{k})$ and $\zeta(\mathbf{l})$, we split the summation domain of the product $\zeta(\mathbf{k}) \zeta(\mathbf{l})$

$$
\left\{0<n_{1}<\ldots<n_{p}\right\} \times\left\{0<m_{1}<\ldots<m_{q}\right\}
$$

into all the domains that preserve the order of the $n_{i}$ as well as the order of the $m_{j}$ and into the boundary domains where some $n_{i}$ are equal to some $m_{j}$. We obtain the following well-known proposition, giving the quadratic relations (2) between multiple zeta values known as the stuffle relations:

Proposition 1.1. Let $\mathbf{k}=\left(k_{1}, \ldots, k_{p}\right)$ and $\mathbf{l}=\left(l_{1}, \ldots, l_{q}\right)$ as above with $k_{1}, l_{1} \geqslant 2$. Then we have:

$$
\zeta(\mathbf{k}) \zeta(\mathbf{l})=\left(\sum_{n_{1}>\ldots>n_{p}>0} \frac{1}{n_{1}^{k_{1}} \cdots n_{p}^{k_{p}}}\right)\left(\sum_{m_{1}>\ldots>m_{q}>0} \frac{1}{m_{1}^{l_{1}} \cdots m_{q}^{l_{q}}}\right)=\sum_{\sigma \in \operatorname{st}(\mathbf{k}, \mathbf{l})} \zeta(\sigma) .
$$

1.2. Integral representation of the shuffle relations. To the tuple $\mathbf{k}$, with $n=k_{1}+\cdots+k_{p}$, we associate the $n$-tuple:

$$
\bar{k}=(\underbrace{0, \ldots, 0}_{k_{1}-1 \text { times }}, 1, \ldots, \underbrace{0, \ldots, 0}_{k_{p}-1 \text { times }}, 1)=\left(\varepsilon_{n}, \ldots, \varepsilon_{1}\right)
$$

and the differential form, introduced by Kontsevich

$$
\omega_{\mathbf{k}}=\omega_{\bar{k}}=(-1)^{p} \frac{d t_{1}}{t_{1}-\varepsilon_{1}} \wedge \cdots \wedge \frac{d t_{n}}{t_{n}-\varepsilon_{n}} .
$$

Then, setting $\Delta_{n}=\left\{0<t_{1}<\ldots<t_{n}<1\right\}$, direct integration yields:

$$
\zeta(\mathbf{k})=\int_{\Delta_{n}} \omega_{\mathbf{k}}
$$

The shuffle product of an $n$-tuple $\left(e_{1}, \ldots, e_{n}\right)=e_{1} \cdot \bar{e}$ and an $m$-tuple $\left(f_{1}, \ldots, f_{m}\right)=$ $f_{1} \cdot \bar{f}$ is defined recursively by:

$$
\left(e_{1}, \ldots, e_{n}\right) \amalg\left(f_{1}, \ldots, f_{m}\right)=e_{1} \cdot\left(\bar{e} \amalg\left(f_{1} \cdot \bar{f}\right)\right)+f_{1} \cdot\left(\left(e_{1} \cdot \bar{e}\right) \amalg \bar{f}\right)
$$

and $\bar{e} \amalg()=() \amalg \bar{e}=\bar{e}$. Here, as above, the + is a formal sum, $b \cdot B$ means that we concatenate $b$ at the beginning of the tuple $B$ and - is linear in $B$.

Let $\mathbf{k}$ and $\mathbf{l}$ be two tuples of integers as above. We will write $\operatorname{sh}(\bar{k}, \bar{l})$ for the set of the individual terms in the formal sum $\bar{k}$ ш $\bar{l}$ whose coefficients are all equal 
to 1 . Such a generic term is then denoted by $\sigma \in \operatorname{sh}(\bar{k}, \bar{l})$ and can be identified with a unique permutation $\tilde{\sigma}$ of $\{1, \ldots n+m\}$ such that $\tilde{\sigma}(1)<\ldots<\tilde{\sigma}(n)$ and $\tilde{\sigma}(n+1)<\ldots<\tilde{\sigma}(n+m)$. The permutation $\tilde{\sigma}$ will simply be denoted by $\sigma$ when the context will be clear enough.

We will put an index $\sigma$ on any object which naturally depends on a shuffle. The following proposition yields the quadratic relations (5) known as the shuffle relations.

Proposition 1.2. Let $\mathbf{k}=\left(k_{1}, \ldots, k_{p}\right)$ and $\mathbf{l}=\left(l_{1}, \ldots, l_{q}\right)$ with $k_{1}, l_{1} \geqslant 2$. Then:

$$
\int_{\Delta_{n}} \omega_{\bar{k}} \int_{\Delta_{m}} \omega_{\bar{l}}=\sum_{\sigma \in \operatorname{sh}(\bar{k}, \bar{l})} \int_{\Delta_{n+m}} \omega_{\sigma} .
$$

Proof. Let $n=k_{1}+\ldots+k_{p}$ and $m=l_{1}+\ldots+l_{q}$. Then we have:

$$
\begin{aligned}
\int_{\Delta_{n}} \omega_{\bar{k}} \int_{\Delta_{m}} \omega_{\bar{l}} & =\left(\int_{\Delta_{n}} \frac{d t_{1}}{1-t_{1}} \cdots \frac{d t_{n}}{t_{n}}\right)\left(\int_{\Delta_{m}} \frac{d t_{n+1}}{1-t_{n+1}} \cdots \frac{d t_{n+m}}{t_{n+m}}\right) \\
& =\int_{\Delta} \frac{d t_{1}}{1-t_{1}} \cdots \frac{d t_{n}}{t_{n}} \frac{d t_{n+1}}{1-t_{n+1}} \cdots \frac{d t_{n+m}}{t_{n+m}} .
\end{aligned}
$$

The set $\Delta=\left\{0<t_{1}<\ldots<t_{n}<1\right\} \times\left\{0<t_{n+1}<\ldots<t_{n+m}<1\right\}$ can be, up to codimension 1 sets, split into a union of simplices

$$
\coprod_{\sigma \in \operatorname{sh}(\llbracket 1, n \rrbracket \llbracket \llbracket n+1, m \rrbracket)} \Delta_{\sigma} \quad \text { with } \Delta_{\sigma}=\left\{0<t_{\sigma(1)}<t_{\sigma(2)}<\ldots<t_{\sigma(n+m)}<1\right\},
$$

where $\llbracket a, b \rrbracket$ denotes the ordered sequence of integers from $a$ to $b$.

The integral over $\Delta$ is the sum of the integrals over the individual simplices. But the integral over one of these simplices is, up to the numbering of the variables, exactly one term of the sum $\sum_{\sigma \in \operatorname{sh}(\bar{k}, \bar{l})} \int_{\Delta_{n+m}} \omega_{\sigma}$.

1.3. The stuffle relations in terms of integrals. We explain here ideas already written in articles of Goncharov [Gon02] and in Francis Brown's Ph.D. thesis [Bro06], showing how to express the stuffle relations (2) in terms of integrals.

1.3.1. Example. We have $\zeta(2)=\int_{\Delta_{2}} \frac{d t_{2}}{t_{2}} \frac{d t_{1}}{1-t_{1}}$. The change of variables $t_{2}=x_{1}$ and $t_{1}=x_{1} x_{2}$ gives:

$$
\zeta(2)=\int_{[0,1]^{2}} \frac{d x_{1}}{x_{1}} \frac{x_{1} d x_{2}}{1-x_{1} x_{2}}=\int_{[0,1]^{2}} \frac{d x_{1} d x_{2}}{1-x_{1} x_{2}} .
$$

This change of variables is nothing but the blow-up of the point $(0,0)$ in the projective plane, given in $n$ dimensions by a sequence of blow-ups:

$$
t_{n}=x_{1}, t_{n-1}=x_{1} x_{2}, \ldots, t_{1}=x_{1} \ldots x_{n} .
$$

We will write $d^{n} x$ for $d x_{1} \cdots d x_{n}$ where $n$ is the number of variables under the integral. Using the change of variables (6) for $n=4$ we write the Kontsevich forms as follows:

$$
\zeta(4)=\int_{[0,1]^{4}} \frac{d^{4} x}{1-x_{1} x_{2} x_{3} x_{4}}, \quad \zeta(2,2)=\int_{[0,1]^{4}} \frac{x_{1} x_{2} d^{4} x}{\left(1-x_{1} x_{2}\right)\left(1-x_{1} x_{2} x_{3} x_{4}\right)}
$$

and

$$
\zeta(2) \zeta(2)=\int_{[0,1]^{4}} \frac{1}{\left(1-x_{1} x_{2}\right)} \frac{1}{\left(1-x_{3} x_{4}\right)} d^{4} x .
$$

For any variables $\alpha$ and $\beta$ we have the equality:

$$
\frac{1}{(1-\alpha)(1-\beta)}=\frac{\alpha}{(1-\alpha)(1-\alpha \beta)}+\frac{\beta}{(1-\beta)(1-\beta \alpha)}+\frac{1}{1-\alpha \beta} \text {. }
$$


This identity will be the key of this section.

Setting $\alpha=x_{1} x_{2}$ and $\beta=x_{3} x_{4}$ and applying (7), we recover the stuffle relation:

$$
\begin{gathered}
\zeta(2) \zeta(2)=\int_{[0,1]^{4}}\left(\frac{x_{1} x_{2}}{\left(1-x_{1} x_{2}\right)\left(1-x_{1} x_{2} x_{3} x_{4}\right)}+\frac{x_{3} x_{4}}{\left(1-x_{3} x_{4}\right)\left(1-x_{3} x_{4} x_{1} x_{2}\right)}\right. \\
\left.+\frac{1}{1-x_{1} x_{2} x_{3} x_{4}}\right) d^{4} x \\
\zeta(2) \zeta(2)=\zeta(2,2)+\zeta(2,2)+\zeta(4) .
\end{gathered}
$$

1.3.2. General case. We will show that the Cartier decomposition (9) below makes it possible to express all the stuffle relations in terms of integrals as in the example above.

Let $\mathbf{k}=\left(k_{1}, \ldots, k_{p}\right)$ and $\mathbf{l}=\left(l_{1}, \ldots, l_{q}\right)$ be two tuples of integers with $k_{1}, l_{1} \geqslant 2$. As above, if $\sigma$ is a term of the formal sum $\mathbf{k} * \mathbf{l}$, we will write $\sigma \in \operatorname{st}(\mathbf{k}, \mathbf{l})$. We will put an index $\sigma$ on any object which naturally depends on a stuffle.

Let $\mathbf{k}=\left(k_{1}, \ldots, k_{p}\right)$ be as above and $n=k_{1}+\cdots+k_{p}$. We define $f_{k_{1}, \ldots, k_{p}}$ to be the function of $n$ variables defined on $[0,1]^{n}$ given by:

$$
\begin{aligned}
& f_{k_{1}, \ldots, k_{p}}\left(x_{1}, \ldots, x_{n}\right)=\frac{1}{1-x_{1} \cdots x_{k_{1}}} \frac{x_{1} \cdots x_{k_{1}}}{1-x_{1} \cdots x_{k_{1}} x_{k_{1}+1} \cdots x_{k_{1}+k_{2}}} \\
& \frac{x_{1} \cdots x_{k_{1}+k_{2}}}{1-x_{1} \cdots x_{k_{1}+k_{2}+k_{3}}} \cdots \frac{x_{1} \cdots x_{k_{1}+\ldots+k_{p-1}}}{1-x_{1} \cdots x_{k_{1}+\cdots+k_{p}}} .
\end{aligned}
$$

Proposition 1.3. For all p-tuples of integers $\left(k_{1}, \ldots, k_{p}\right)$ with $k_{1} \geqslant 2$, we have (with $n=k_{1}+\cdots+k_{p}$ ):

$$
\zeta\left(k_{1}, \ldots, k_{p}\right)=\int_{[0,1]^{n}} f_{k_{1}, \ldots, k_{p}}\left(x_{1}, \ldots, x_{n}\right) d^{n} x .
$$

Proof. Let $\omega_{\mathbf{k}}$ be the Kontsevich form associated to a $p$-tuple $\left(k_{1}, \ldots, k_{p}\right)$ with $n=k_{1}+\cdots+k_{p}$, so that $\zeta\left(k_{1}, \ldots, k_{p}\right)=\int_{\Delta_{n}} \omega_{\mathbf{k}}$.

Applying the change of variables (6) to $\omega_{\mathbf{k}}$, we see that for each term $\frac{d t_{i}}{t_{i}}$, there arises from the $\frac{1}{t_{i}}$ a term $\frac{1}{x_{1} \cdots x_{n-i+1}}$ which cancels with $\frac{d t_{i-1}}{\cdots}=\frac{x_{1} \cdots x_{n-i+1} d x_{n-i+2}}{\cdots}$. This gives the result.

To derive the stuffle relations in general using integrals and the functions $f_{k_{1}, \ldots, k_{p}}$, we will use the following notation.

Notation. Let $\mathbf{k}$ be a sequence $\left(k_{1}, \ldots, k_{p}\right), n=k_{1}+\cdots+k_{p}$. We have $n$ variables $x_{1}, \ldots, x_{n}$.

- For any sequence $\mathbf{a}=\left(a_{1}, \ldots, a_{r}\right)$, we will write $\prod \mathbf{a}=a_{1} \cdots a_{r}$.

- The sequence $\left(x_{1}, \ldots, x_{n}\right)$ will be written $\mathbf{x}$. We set $\mathbf{x}(\mathbf{k}, 1)=\left(x_{1}, \ldots, x_{k_{1}}\right)$ and

$$
\mathbf{x}(\mathbf{k}, i)=\left(x_{k_{1}+\cdots+k_{i-1}+1}, \ldots, x_{k_{1}+\cdots+k_{i}}\right)
$$

so the $\mathbf{x}$ is the concatenation of sequences $\mathbf{x}(\mathbf{k}, 1) \cdots \mathbf{x}(\mathbf{k}, p)$.

- The sequence $\left(x_{1}, \ldots, x_{k_{1}+\cdots+k_{i}}\right)=\mathbf{x}(\mathbf{k}, 1) \cdots \mathbf{x}(\mathbf{k}, i)$ will be denoted by $\mathbf{x}(\mathbf{k}, \leqslant i)$. If $\mathbf{k}=\left(\mathbf{k}_{\mathbf{0}}, k_{p}\right), \mathbf{x}_{0}=\mathbf{x}(\mathbf{k}, \leqslant p-1)$ will be the sequence

$$
\left(x_{1}, \ldots, x_{k_{1}+\cdots+k_{p-1}}\right) \text {. }
$$

- If $\mathbf{l}$ is a $q$-tuple with $l_{1}+\cdots+l_{q}=m$ and $\sigma \in \operatorname{st}(\mathbf{k}, \mathbf{l}), y_{\sigma}$ will be the sequence in the variables $x_{1}, \ldots, x_{n}, x_{1}^{\prime}, \ldots, x_{m}^{\prime}$ in which each group of variables

$$
\begin{gathered}
\mathbf{x}(\mathbf{k}, i)=\left(x_{k_{1}+\cdots+k_{i-1}+1}, \ldots, x_{k_{1}+\cdots+k_{i}}\right) \\
\left(\operatorname{resp} . \mathbf{x}^{\prime}(\mathbf{l}, j)=\left(x_{l_{1}+\cdots+l_{j-1}+1}^{\prime}, \ldots, x_{l_{1}+\cdots+l_{j}}^{\prime}\right)\right)
\end{gathered}
$$


is in the position of $k_{i}$ (resp. $l_{j}$ ) in $\sigma$. Components of $\sigma$ of the form $k_{i}+l_{j}$ give rise to subsequences like

$$
\left(x_{k_{1}+\cdots+k_{i-1}+1}, \ldots, x_{k_{1}+\cdots+k_{i}}, x_{l_{1}+\cdots+l_{j-1}+1}^{\prime}, \ldots, x_{l_{1}+\cdots+l_{j}}^{\prime}\right)=\left(\mathbf{x}(\mathbf{k}, i), \mathbf{x}^{\prime}(\mathbf{l}, j)\right) .
$$

- Following these notations, products $x_{1} \cdots x_{k_{1}}, x_{k_{1}+\cdots+k_{i-1}+1} \cdots x_{k_{1}+\cdots+k_{i}}$, $x_{1} \cdots x_{k_{1}+\cdots+k_{i}}$ will be written respectively $\prod \mathbf{x}(\mathbf{k}, 1), \prod \mathbf{x}(\mathbf{k}, i), \prod \mathbf{x}(\mathbf{k}, \leqslant$ $i)$. As $\mathbf{x}(\mathbf{k}, \leqslant p-1)=\mathbf{x}_{0}$ and $\mathbf{x}(\mathbf{k}, \leqslant p)=\mathbf{x}$, products $\prod \mathbf{x}(\mathbf{k}, \leqslant p-1)$ and $\prod \mathbf{x}(\mathbf{k}, \leqslant p)$ will be written $\prod \mathbf{x}_{0}$ and $\prod \mathbf{x}$.

We remark that for each $\sigma \in \operatorname{st}(\mathbf{k}, \mathbf{l}), \prod \sigma=\prod \mathbf{x} \prod \mathbf{x}^{\prime}$.

Remark 1.4. Let $\left(k_{1}, \ldots, k_{p}\right)=\left(\mathbf{k}_{\mathbf{0}}, k_{p}\right)$ be a sequence of integers. Then:

$$
f_{k_{1}, \ldots, k_{p}}(\mathbf{x})=f_{k_{1}, \ldots, k_{p-1}}(\mathbf{x}(\mathbf{k}, \leqslant p-1)) \frac{\prod \mathbf{x}(\mathbf{k}, \leqslant p-1)}{1-\prod \mathbf{x}(\mathbf{k}, \leqslant p)}=f_{k_{1}, \ldots, k_{p-1}}\left(\mathbf{x}_{0}\right) \frac{\prod \mathbf{x}_{0}}{1-\prod \mathbf{x}} .
$$

Proposition 1.5. Let $\mathbf{k}=\left(k_{1}, \ldots, k_{p}\right)$ and $\mathbf{l}=\left(l_{1}, \ldots, l_{q}\right)$ be two sequences of weight $n$ and $m$. Then:

(9) $f_{k_{1}, \ldots, k_{p}}(\mathbf{x}(\mathbf{k}, 1), \ldots, \mathbf{x}(\mathbf{k}, p)) \cdot f_{l_{1}, \ldots, l_{q}}\left(\mathbf{x}^{\prime}(\mathbf{l}, 1), \ldots, \mathbf{x}^{\prime}(\mathbf{l}, q)\right)=\sum_{\sigma \in \mathbf{s t}(\mathbf{k}, \mathbf{l})} f_{\sigma}\left(y_{\sigma}\right)$.

Proof. We proceed by induction on the depth of the sequence. The recursion formula for the stuffle is given in (1).

If $p=q=1$ : As we have

$$
f_{n}(\mathbf{x}(\mathbf{k}, 1)) f_{m}\left(\mathbf{x}^{\prime}(\mathbf{l}, 1)\right)=\frac{1}{1-\prod \mathbf{x}(\mathbf{k}, \leqslant 1)} \cdot \frac{1}{1-\prod \mathbf{x}^{\prime}(\mathbf{l}, \leqslant 1)}=\frac{1}{1-\prod \mathbf{x}} \cdot \frac{1}{1-\prod \mathbf{x}^{\prime}}
$$

using the formula (7) with $\alpha=\prod \mathbf{x}$ and $\beta=\prod \mathbf{x}^{\prime}$ leads to

$$
\begin{array}{r}
f_{n}(\mathbf{x}(\mathbf{k}, 1)) f_{m}\left(\mathbf{x}^{\prime}(\mathbf{l}, 1)\right)=\frac{\prod \mathbf{x}}{\left(1-\prod \mathbf{x}\right)\left(1-\prod \mathbf{x} \prod \mathbf{x}^{\prime}\right)}+\frac{\prod \mathbf{x}^{\prime}}{\left(1-\prod \mathbf{x}^{\prime}\right)\left(1-\prod \mathbf{x}^{\prime} \prod \mathbf{x}\right)} \\
+\frac{1}{1-\prod \mathbf{x} \prod \mathbf{x}^{\prime}} .
\end{array}
$$

Inductive step: Let $\left(k_{1}, \ldots, k_{p}\right)=\left(\mathbf{k}_{\mathbf{0}}, k_{p}\right)$ and $\left(l_{1}, \ldots, l_{q}\right)=\left(\mathbf{l}_{\mathbf{0}}, l_{q}\right)$ be two sequences. By Remark 1.4, the following equality holds

$$
f_{\mathbf{k}_{0}, k_{p}}\left(\mathbf{x}_{0}, \mathbf{x}(\mathbf{k}, p)\right) f_{\mathbf{l}_{\mathbf{0}}, l_{q}}\left(\mathbf{x}_{0}{ }^{\prime}, \mathbf{x}^{\prime}(\mathbf{l}, q)\right)=f_{\mathbf{k}_{\mathbf{0}}}\left(\mathbf{x}_{0}\right) \frac{\prod \mathbf{x}_{0}}{1-\prod \mathbf{x}} f_{\mathbf{l}_{\mathbf{0}}}\left(\mathbf{x}_{0}{ }^{\prime}\right) \frac{\prod \mathbf{x}_{0}^{\prime}}{1-\prod \mathbf{x}^{\prime}} .
$$

Applying the formula (7) with $\alpha=\prod \mathbf{x}$ and $\beta=\prod \mathbf{x}^{\prime}$, one sees that the RHS of the previous equation is equal to

$$
\begin{aligned}
& f_{\mathbf{k}_{\mathbf{0}}}\left(\mathbf{x}_{0}\right) f_{\mathbf{l}_{\mathbf{0}}}\left(\mathbf{x}_{0}{ }^{\prime}\right) \cdot\left(\prod \mathbf{x}_{0} \cdot \prod \mathbf{x}_{0}^{\prime}\right)\left(\frac{\prod \mathbf{x}}{\left(1-\prod \mathbf{x}\right)\left(1-\prod \mathbf{x} \prod \mathbf{x}^{\prime}\right)}\right. \\
&\left.+\frac{\prod \mathbf{x}^{\prime}}{\left(1-\prod \mathbf{x}^{\prime}\right)\left(1-\prod \mathbf{x}^{\prime} \prod \mathbf{x}\right)}+\frac{1}{\left(1-\prod \mathbf{x} \prod \mathbf{x}^{\prime}\right)}\right) .
\end{aligned}
$$


Expanding and using Remark 1.4 we obtain:

$$
\begin{aligned}
& f_{\mathbf{k}_{\mathbf{0}}, k_{p}}\left(\mathbf{x}_{0}, \mathbf{x}(\mathbf{k}, p)\right) f_{\mathbf{l}_{\mathbf{o}}, l_{q}}\left(\mathbf{x}_{0}{ }^{\prime}, \mathbf{x}^{\prime}(\mathbf{l}, q)\right)= \\
& \left(f_{\mathbf{k}_{\mathbf{0}}, k_{p}}(\mathbf{x}) f_{\mathbf{l}_{\mathbf{o}}}\left(\mathbf{x}_{0}{ }^{\prime}\right)\right) \cdot \frac{\prod \mathbf{x} \prod \mathbf{x}_{0}^{\prime}}{1-\prod \mathbf{x} \prod \mathbf{x}^{\prime}}+\left(f_{\mathbf{k}_{\mathbf{0}}}\left(\mathbf{x}_{0}\right) f_{\mathbf{l}_{\mathbf{o}}, l_{q}}\left(\mathbf{x}^{\prime}\right)\right) \cdot \frac{\prod \mathbf{x}^{\prime} \prod \mathbf{x}_{0}}{1-\prod \mathbf{x}^{\prime} \prod \mathbf{x}} \\
& +\left(f_{\mathbf{k}_{\mathbf{0}}}\left(\mathbf{x}_{0}\right) f_{\mathbf{l}_{\mathbf{o}}}\left(\mathbf{x}_{0}{ }^{\prime}\right)\right) \cdot \frac{\prod \mathbf{x}_{0} \prod \mathbf{x}_{0}^{\prime}}{1-\prod \mathbf{x} \prod \mathbf{x}^{\prime}} .
\end{aligned}
$$

Hence, the product of functions $f_{k_{1}, \ldots, k_{p}}$ and $f_{l_{1}, \ldots, l_{q}}$ satisfies a recursion formula identical to the formula (1) defining the stuffle product. Using induction, the proposition follows.

Corollary 1.6 (integral representation of the stuffle). Integrating the statement of the previous proposition over the cube and permuting the variables in each term of the RHS, we obtain:

$\zeta(\mathbf{k}) \zeta(\mathbf{l})=\int_{[0,1]^{n}} f_{\mathbf{k}} d^{n} x \int_{[0,1]^{m}} f_{\mathbf{l}} d^{m} x=\int_{[0,1]^{n+m}} \sum_{\sigma \in \mathbf{s t}(\mathbf{k}, \mathbf{l})} f_{\sigma} d^{n+m} x=\sum_{\sigma \in \mathbf{s t}(\mathbf{k}, \mathbf{l})} \zeta(\sigma)$.

Proof. We only need to check that all integrals are convergent. As all the functions are positive on the integration domain, all variable changes are allowed, and we can deduce the convergence of each term from the convergence of the iterated integral representation for the multiple zeta values.

Another argument is to remark that the orders of the poles of our functions along a codimension $k$ subvariety can be at most $k$. Then, for each integral, a succession of blow-ups ensures that the integral converges.

\section{Moduli SPACES OF CURVES; DOUBlE ShUfFle AND FORGETFul MAPS}

2.1. Shuffle and moduli spaces of curves. Let $\mathbf{k}$ and $\mathbf{l}$ be as in the previous section, let $n=k_{1}+\cdots+k_{p}$ and $m=l_{1}+\cdots+l_{q}$. Following the article of Goncharov and Manin [GM04], we will identify a point of $\mathcal{M}_{0, j+3}$, the moduli space of curves of genus 0 with $j+3$ marked points, with a sequence $\left(0, z_{1}, \ldots, z_{j}, 1, \infty\right)$, the $z_{i}$ being pairwise distinct and distinct from 0,1 and $\infty$, and write $\Phi_{j}$ for the open cell in $\mathcal{M}_{0, j+3}(\mathbb{R})$ which is mapped onto $\Delta_{j}$, the standard simplex, by the map: $\mathcal{M}_{0, j+3} \rightarrow\left(\mathbb{P}^{1}\right)^{j},\left(0, z_{1}, \ldots, z_{j}, 1, \infty\right) \mapsto\left(z_{1}, \ldots, z_{j}\right)$. Then we have:

$$
\zeta\left(k_{1}, \ldots, k_{p}\right)=\int_{\Phi_{n}} \omega_{\mathbf{k}} .
$$

Proposition 2.1. Let $\beta$ be the map defined by

$$
\begin{array}{ccc}
\mathcal{M}_{0, n+m+3} & \stackrel{\beta}{\longmapsto} & \mathcal{M}_{0, n+3} \times \mathcal{M}_{0, m+3} \\
\left(0, z_{1}, \ldots, z_{n+m}, 1, \infty\right) & \longmapsto & \left(0, z_{1}, \ldots, z_{n}, 1, \infty\right) \times\left(0, z_{n+1}, \ldots, z_{n+m}, 1, \infty\right) .
\end{array}
$$

Then, letting $t_{i}$ be the coordinate such that $t_{i}\left(0, z_{1}, \ldots, z_{n+m}, 1, \infty\right)=z_{i}$, we have

$$
\beta^{*}\left(\omega_{\mathbf{k}} \wedge \omega_{\mathbf{l}}\right)=\frac{d t_{1}}{1-t_{1}} \wedge \cdots \wedge \frac{d t_{n}}{t_{n}} \wedge \frac{d t_{n+1}}{1-t_{n+1}} \wedge \cdots \wedge \frac{d t_{n+m}}{t_{n+m}} .
$$

Furthermore, if for $\sigma \in \operatorname{sh}(\llbracket 1, n \rrbracket, \llbracket n+1, n+m \rrbracket)$ we write $\Phi_{n+m}^{\sigma}$ for the open cell of $\mathcal{M}_{0, n+m+3}(\mathbb{R})$ in which the points are in the same order as their indices are in $\sigma$, we have

$$
\beta^{-1}\left(\Phi_{n} \times \Phi_{m}\right)=\coprod_{\sigma \in \operatorname{sh}(\llbracket 1, n \rrbracket, \llbracket n+1, n+m \rrbracket)} \Phi_{n+m}^{\sigma}
$$


Proof. The first part is obvious.

In order to show that $\beta^{-1}\left(\Phi_{n} \times \Phi_{m}\right)=\coprod \Phi_{n+m}^{\sigma}$ we have to remember that a cell in $\mathcal{M}_{0, n+m+3}(\mathbb{R})$ is given by a cyclic order on the marked points. Let $X=$ $\left(0, z_{1}, \ldots, z_{n+m}, 1, \infty\right)$ be a point in $\mathcal{M}_{0, n+m+3}(\mathbb{R})$ such that $\beta(X) \in \Phi_{n} \times \Phi_{m}$. The values of the $z_{i}$ have to be such that

$$
0<z_{1}<\ldots<z_{n}<1(<\infty) \text { and } 0<z_{n+1}<\ldots<z_{n+m}<1(<\infty) .
$$

However there is no order condition relating, say $z_{1}$ to $z_{n+1}$.

So, points on $\mathcal{M}_{0, n+m+3}(\mathbb{R})$ which are in $\beta^{-1}\left(\Phi_{n} \times \Phi_{m}\right)$ are such that the $z_{i}$ are compatible with (12). That is, they are in $\coprod_{\sigma \in \operatorname{sh}(\llbracket 1, n \rrbracket, \llbracket n+1, n+m \rrbracket)} \Phi_{n+m}^{\sigma}$.

As $\Phi_{n} \times \Phi_{m} \backslash\left(\beta\left(\beta^{-1}\left(\Phi_{n} \times \Phi_{m}\right)\right)\right)$ is of codimension 1 , we have the following proposition.

Proposition 2.2. The shuffle relation $\zeta(\mathbf{k}) \zeta(\mathbf{l})=\sum_{\sigma \in \operatorname{sh}(\mathbf{k}, \mathbf{l})} \zeta(\sigma)$ is a consequence of the following change of variables:

$$
\int_{\Phi_{n} \times \Phi_{m}} \omega_{\mathbf{k}} \wedge \omega_{\mathbf{l}}=\int_{\beta^{-1}\left(\Phi_{n} \times \Phi_{m}\right)} \beta^{*}\left(\omega_{\mathbf{k}} \wedge \omega_{\mathbf{l}}\right) .
$$

Proof. Using the previous proposition, the right hand side of this equality is equal to

$$
\sum_{\sigma \in \operatorname{sh}(\llbracket 1, n \rrbracket, \llbracket n+1, n+m \rrbracket)} \int_{\Phi_{n+m}^{\sigma}} \frac{d t_{1}}{1-t_{1}} \wedge \cdots \wedge \frac{d t_{n+m}}{t_{n+m}} .
$$

Then we permute the variables and change their names in order to have an integral over $\Phi_{n+m}$ for each term. This is the same computation we did for the integral over $\mathbb{R}^{n+m}$ in proposition 1.2.

As the form $\frac{d t_{1}}{1-t_{1}} \wedge \cdots \wedge \frac{d t_{n+m}}{t_{n+m}}$ (resp. $\frac{d t_{\sigma(1)}}{1-t_{\sigma(1)}} \wedge \cdots \wedge \frac{d t_{\sigma(n+m)}}{t_{\sigma(n+m)}}$ ) does not have any poles on the boundary of $\Phi_{n+m}^{\sigma}$ (resp. $\left.\Phi_{n+m}\right)$, all the integrals are convergent.

2.2. Stuffle and moduli spaces of curves. In Section 1.3, in order to have an integral representation of the stuffle product, we recalled, using the integral over a simplex and a change of variables, a cubical representation of the MZVs (integral over a cube). We use here a similar change of variables to introduce another system of local coordinates on $\overline{\mathcal{M}_{0, r+3}}$, the Deligne-Mumford compactification of the moduli space of curves. Following [Bro06], we will speak of cubical coordinates. Those cubical coordinates, $u_{i}$, are defined on an open subset of $\overline{\mathcal{M}_{0, r+3}}$ by $u_{1}=t_{r}$ and $u_{i}=t_{r-i+1} / t_{r-i+2}$ for $i<r$ where the $t_{i}$ are the usual (simplicial) coordinates on $\overline{\mathcal{M}_{0, r+3}}$. This cubical system is well adapted to express the stuffle relations on the moduli spaces of curves.

Proposition 2.3. Let $\delta$ be the map defined by

$$
\begin{aligned}
\mathcal{M}_{0, n+m+3} & \stackrel{\delta}{ } \mathcal{M}_{0, n+3} \times \mathcal{M}_{0, m+3} \\
\left(0, z_{1}, \ldots, z_{n+m}, 1, \infty\right) & \longmapsto\left(0, z_{m+1}, \ldots, z_{m+n}, 1, \infty\right) \times\left(0, z_{1}, \ldots, z_{m}, z_{m+1}, \infty\right) .
\end{aligned}
$$

Writing the expression of $\omega_{\mathbf{k}}$ and $\omega_{\mathbf{l}}$ in cubical coordinates, one finds $\omega_{\mathbf{k}}=$ $f_{\mathbf{k}}\left(u_{1}, \ldots, u_{n}\right) d^{n} u$ and $\omega_{\mathbf{l}}=f_{\mathbf{l}}\left(u_{n+1}, \ldots, u_{n+m}\right) d^{m} u$ where the $f_{\mathbf{k}}$ are as in section 1.3. Then, using those coordinates we have

$$
\delta^{*}\left(\omega_{\mathbf{k}} \wedge \omega_{\mathbf{l}}\right)=f_{k_{1}, \ldots, k_{p}}\left(u_{1}, \ldots, u_{n}\right) f_{l_{1}, \ldots, l_{q}}\left(u_{n+1}, \ldots, u_{n+m}\right) d^{n+m} u
$$

and

$$
\delta^{-1}\left(\Phi_{n} \times \Phi_{m}\right)=\Phi_{n+m}
$$


Proof. To prove the second statement, let $X=\left(0, z_{1}, \ldots, z_{n+m}, 1, \infty\right)$ be such that $\delta(X) \in \Phi_{n} \times \Phi_{m}$. Then the values of the $z_{i}$ 's have to verify

$$
0<z_{1}<\ldots<z_{m}<z_{m+1}(<\infty) \text { and } 0<z_{m+1}<\ldots<z_{n+m}<1(<\infty) \text {. }
$$

These conditions show that $0<z_{1}<\ldots<z_{m}<z_{m+1}<\ldots<1<\infty$, so $X \in \Phi_{n+m}$.

To prove the first statement, we claim that $\delta$ is expressed in cubical coordinates by

$$
\left(u_{1}, \ldots, u_{n+m}\right) \longmapsto\left(u_{1}, \ldots, u_{n}\right) \times\left(u_{n+1}, \ldots, u_{n+m}\right) .
$$

It is obvious to see that for the left hand factor the coordinates are unchanged. For the right hand factor we have to rewrite the expression of the right side in terms of the standard representatives on $\mathcal{M}_{0, m+3}$. We have

$$
\left(0, z_{1}, \ldots, z_{m}, z_{m+1}, \infty\right)=\left(0, z_{1} / z_{m+1}, \ldots, z_{m} / z_{m+1}, 1, \infty\right)=\left(0, t_{1}, \ldots, t_{m}, 1, \infty\right)
$$

in simplicial coordinates. This point is given in cubical coordinates on $\mathcal{M}_{0, m+3}$ by

$$
\left(t_{m}, t_{m-1} / t_{m}, \ldots, t_{1} / t_{2}\right)=\left(z_{m} / z_{m+1}, \ldots, z_{1} / z_{2}\right)=\left(u_{n+1}, \ldots, u_{n+m}\right) .
$$

As a consequence of this discussion and the results of Section 1.3, we have the following proposition.

Proposition 2.4. Using the Cartier decomposition (9), the stuffle product can be viewed as the change of variables:

$$
\int_{\Phi_{n} \times \Phi_{m}} \omega_{\mathbf{k}} \wedge \omega_{\mathbf{l}}=\int_{\delta^{-1}\left(\Phi_{n} \times \Phi_{m}\right)} \delta^{*}\left(\omega_{\mathbf{k}} \wedge \omega_{\mathbf{l}}\right) .
$$

Remark 2.5. We should point out here the fact that the Cartier decomposition "does not lie in the moduli spaces of curves", in the sense that forms appear in the decomposition which are not holomorphic on the moduli space. For example, in the Cartier decomposition of $f_{2,1}\left(u_{1}, u_{2}, u_{3}\right) f_{2,1}\left(u_{4}, u_{5}, u_{6}\right)$, we see the term

$$
\frac{u_{1} u_{2} u_{4} u_{5} d u_{1} d u_{2} d u_{3} d u_{4} d u_{5} d u_{6}}{\left(1-u_{1} u_{2} u_{4} u_{5}\right)\left(1-u_{1} u_{2} u_{3} u_{4} u_{5} u_{6}\right)}
$$

which is not a holomorphic differential form on $\mathcal{M}_{0,6}$. However, it is a well-defined convergent form on the standard cell where it is integrated. Changing the numbering of the variables (which stabilises the standard cell) gives the equality with $\zeta(4,2)$. This example represents the situation in the general case: when simply dealing with integrals, the non-holomorphic forms are not a problem. However, in the context of framed motives, they are.

\section{Motivic Shuffle For The "CONVERGEnT" WORDS}

3.1. Framed mixed Tate motives and motivic multiple zeta values. This section is a short introduction to the motivic tools we will use to prove the motivic double shuffle. The motivic context is a cohomological version of Voevodsky's category $\mathcal{D} \mathcal{M}_{\mathbb{Q}}$ [Voe00]. Goncharov developed in [Gon99], [Gon05] and [Gon01] an additional structure on mixed Tate motives, introduced in [BGSV90], in order to select a specific period of a mixed Tate motive.

An $n$-framed mixed Tate motive is a mixed Tate motive $M$ equipped with two non-zero morphisms:

$$
v: \mathbb{Q}(-n) \rightarrow \operatorname{Gr}_{2 n}^{W} M \quad f: \mathbb{Q}(0) \rightarrow\left(\mathrm{Gr}_{0}^{W} M\right)^{\vee}=\mathrm{Gr}_{0}^{W} M^{\vee}
$$

On the set of all $n$-framed mixed Tate motives, we consider the coarsest equivalence relation under which $(M, v, f) \sim\left(M^{\prime}, v^{\prime}, f^{\prime}\right)$ if there is a linear map $M \rightarrow M^{\prime}$ 
respecting the frames. Let $\mathcal{A}_{n}$ be the set of equivalence classes and $\mathcal{A}_{\bullet}$ the direct sum of the $\mathcal{A}_{n}$. We write $[M ; v ; f]$ for an equivalence class.

Theorem 3.1 ([Gon05]). A. has a natural structure of graded commutative Hopf algebra over $\mathbb{Q}$.

$\mathcal{A}_{\text {. }}$ is canonically isomorphic to the dual of the Hopf algebra of all endomorphisms of the fibre functor of the Tannakian category of mixed Tate motives.

In our context, the morphism $v$ of a frame should be linked with some differential form and the morphism $f$ is a homological counterpart of $v$, that is a real simplex.

We give here two technical lemmas that will be used in the next sections. We write $[M, v, f]$ for the equivalence class of $(M, v, f)$ in $\mathcal{A}_{\bullet}$. By a slight abuse of notation, we will speak of framed mixed Tate motives refering to both $(M, v, f)$ and $[M, v, f]$.

We recall that the addition of two framed mixed Tate motives $[M, v, f]$ and $\left[M^{\prime}, v^{\prime}, f^{\prime}\right]$ is given by

$$
[M, v, f] \oplus\left[M^{\prime}, v^{\prime}, f^{\prime}\right]=\left[M \oplus M^{\prime},\left(v, v^{\prime}\right), f+f^{\prime}\right] .
$$

Lemma 3.2. Let $M$ be a mixed Tate motive. $v, v_{1}, v_{2}: \mathbb{Q}(-n) \rightarrow \mathrm{Gr}_{2 n}^{W} M$ and $f, f_{1}, f_{2}: \mathbb{Q}(0) \rightarrow \mathrm{Gr}_{0}^{W} M^{\vee}$. We have:

$$
\left[M ; v ; f_{1}+f_{2}\right]=\left[M ; v ; f_{1}\right]+\left[M ; v ; f_{2}\right]
$$

and

$$
\left[M ; v_{1}+v_{2} ; f\right]=\left[M ; v_{1} ; f\right]+\left[M ; v_{2} ; f\right] .
$$

Proof. It follows directly from the definition in [Gon05]. For the first case, it is straightforward to check that the diagonal map $\varphi: M \rightarrow M \oplus M$ is compatible with the frames. For the second equality, the map from $M \oplus M$ to $M$ which sends $\left(m_{1}, m_{2}\right)$ to $m_{1}+m_{2}$ gives the map between the underlying vector spaces and respects the frames.

Lemma 3.3. Let $M$ and $M^{\prime}$ be two mixed Tate motives. Let $M$ be framed by $v: \mathbb{Q}(-n) \rightarrow \mathrm{Gr}_{2 n}^{W}$ and $f: \mathbb{Q}(0) \rightarrow \mathrm{Gr}_{0}^{W} M^{\vee}$. Suppose there exists $v^{\prime}: \mathbb{Q}(-n) \rightarrow$ $\operatorname{Gr}_{2 n}^{W} M^{\prime}$ and $\varphi: M^{\prime} \rightarrow M$ compatible with $v$ and $v^{\prime}$. Then $f$ induces a map $f^{\prime}: \mathbb{Q}(0) \rightarrow \operatorname{Gr}_{0}^{W} M^{\prime \vee}$ and if $f^{\prime}$ is non zero, then $\varphi$ gives an equality of framed mixed Tate motives $[M ; v ; f]=\left[M ; v^{\prime} ; f^{\prime}\right]$.

We recall a classical result, used in [GM04] and described more explicitly in [Gon02], that allows us to build mixed Tate motives from natural geometric situations. In [Gon02], A.B. Goncharov defined a Tate variety as a smooth projective variety $\mathcal{M}$ such that the motive of $\mathcal{M}$ is a direct sum of copies of the Tate motive $\mathbb{Q}(m)$ (for certain $m$ ). We say that a divisor $D$ on $\mathcal{M}$ provides a Tate stratification on $\mathcal{M}$ if all strata of $D$, including $D_{\emptyset}=\mathcal{M}$, are Tate varieties.

Let $\mathcal{M}$ be a smooth variety and $X$ and $Y$ be two normal crossing divisors on $\mathcal{M}$. Let $Y^{X}$ denote $Y \backslash(Y \cap X)$, which is a normal crossing divisor on $\mathcal{M} \backslash X$.

Lemma 3.4. Let $\mathcal{M}$ be a smooth variety of dimension $n$ over $\mathbb{Q}$ and $X \cup Y$ be a normal crossing divisor on $\mathcal{M}$ providing a Tate stratification of $\mathcal{M}$. If $X$ and $Y$ share no common irreducible components then there exists a mixed Tate motive:

$$
\mathrm{H}^{n}\left(\mathcal{M} \backslash X ; Y^{X}\right)
$$

such that its different realisations are given by the respective relative cohomology groups.

We have the following version given in [GM04]. 
Corollary 3.5. Let $X$ and $Y$ be two normal crossing divisors on $\partial \overline{\mathcal{M}_{0, n+3}}$ and suppose they do not share any irreducible components. Then, any choice of nonzero elements

$$
\left[\omega_{X}\right] \in \operatorname{Gr}_{2 n}^{W}\left(\mathrm{H}^{n}\left(\overline{\mathcal{M}_{0, n+3}} \backslash X\right)\right) ; \quad\left[\Phi_{Y}\right] \in \operatorname{Gr}_{0}^{W}\left(\mathrm{H}^{n}\left(\overline{\mathcal{M}_{0, n+3}} ; Y\right)\right)^{\vee}
$$

defines a framed mixed Tate motive given by

$$
\left[\mathrm{H}^{n}\left(\overline{\mathcal{M}_{0, n+3}} \backslash X ; Y^{X}\right) ;\left[\omega_{X}\right] ;\left[\Phi_{Y}\right]\right] .
$$

The following lemma shows that we have some flexibility in choosing $X$ and $Y$ for the framed mixed Tate motive $\left[\mathrm{H}^{n}\left(\mathcal{M} \backslash X ; Y^{X}\right) ;\left[\omega_{X}\right] ;\left[\Phi_{Y}\right]\right]$.

Lemma 3.6. With the notation of Lemma 3.4, let $X^{\prime}$ be a normal crossing divisor containing $X$ which still does not share any irreducible component with $Y, X^{\prime} \cup Y$ being a normal crossing divisor. Then:

$$
\left[\mathrm{H}^{n}\left(\mathcal{M} \backslash X ; Y^{X}\right) ;\left[\omega_{X}\right] ;\left[\Phi_{Y}\right]\right]=\left[\mathrm{H}^{n}\left(\mathcal{M} \backslash X^{\prime} ; Y^{X^{\prime}}\right) ;\left[\omega_{X}\right] ;\left[\Phi_{Y}\right]\right] .
$$

Suppose now that $Y^{\prime}$ is a normal crossing divisor containing $Y$ which does not share any irreducible component with $X^{\prime}, X^{\prime} \cup Y^{\prime}$ being a normal crossing divisor. Then:

$$
\left[\mathrm{H}^{n}\left(\mathcal{M} \backslash X^{\prime} ; Y^{X^{\prime}}\right) ;\left[\omega_{X}\right] ;\left[\Phi_{Y}\right]\right]=\left[\mathrm{H}^{n}\left(\mathcal{M} \backslash X^{\prime} ; Y^{\prime X^{\prime}}\right) ;\left[\omega_{X}\right] ;\left[\Phi_{Y}\right]\right] .
$$

We are now in a position to introduce Goncharov and Manin's definition of motivic multiple zeta values.

Definition 3.7. In particular, let $\mathbf{k}$ be a $p$-tuple with $k_{1} \geqslant 2$ and let $A_{\mathbf{k}}$ be the divisor of singularities of $\omega_{\mathbf{k}}$. Let $B_{n}$ be the Zariski closure of the boundary of $\Phi_{n}$. The motivic multiple zeta value is defined in [GM04] by:

$$
\left[\mathrm{H}^{n}\left(\overline{\mathcal{M}_{0, n+3}} \backslash A_{\mathbf{k}} ; B_{n}^{A_{\mathbf{k}}}\right) ;\left[\omega_{\mathbf{k}}\right] ;\left[\Phi_{n}\right]\right] .
$$

3.2. Motivic Shuffle. The map $\beta$ defined in Proposition 2.1 will be the key to check that the motivic multiple zeta values satisfy the shuffle relations. This map extends continuously to the Deligne-Mumford compactification of the moduli spaces of curves:

$$
\overline{\mathcal{M}_{0, n+m+3}} \stackrel{\beta}{\longrightarrow} \overline{\mathcal{M}_{0, n+3}} \times \overline{\mathcal{M}_{0, m+3}} \text {. }
$$

Let $\omega_{\mathbf{k}}$ and $\omega_{\mathbf{l}}$ be as in section 2.1 , and write $A_{\mathbf{k}}$ and $A_{\mathbf{l}}$ for their respective divisors of singularities. Let $B_{n}$ and $B_{m}$ denote the Zariski closures of the boundary of $\Phi_{n}$ and $\Phi_{m}$ respectively. For $\sigma \in \operatorname{sh}(\llbracket 1, n \rrbracket, \llbracket n+1, n+m \rrbracket)$, let $\omega_{\sigma}$ denote the differential form which corresponds to the shuffled MZV and let $A_{\sigma}$ denote its divisor of singularities. Let $B_{n+m}$ denote the Zariski closure of the boundary of $\Phi_{n+m}$ and $B_{\sigma}$ that of $\Phi_{n+m}^{\sigma}$. The shuffle relations between motivic multiple zeta values are given in the following proposition.

Proposition 3.8. We have an equality of framed motives:

$$
\begin{array}{r}
{\left[\mathrm{H}^{n}\left(\overline{\mathcal{M}_{0, n+3}} \backslash A_{\mathbf{k}} ; B_{n}^{A_{\mathbf{k}}}\right) ;\left[\omega_{\mathbf{k}}\right] ;\left[\Phi_{n}\right]\right] \cdot\left[\mathrm{H}^{m}\left(\overline{\mathcal{M}_{0, m+3}} \backslash A_{\mathbf{l}} ; B_{m}^{A_{\mathbf{l}}}\right) ;\left[\omega_{\mathbf{l}}\right] ;\left[\Phi_{m}\right]\right]=} \\
\sum_{\sigma \in \operatorname{sh}(\llbracket 1, n \rrbracket, \llbracket n+1, n+m \rrbracket)}\left[\mathrm{H}^{n+m}\left(\overline{\mathcal{M}_{0, n+m+3}} \backslash A_{\sigma} ; B_{n+m}^{A_{\sigma}}\right) ;\left[\omega_{\sigma}\right] ;\left[\Phi_{n+m}\right]\right] .
\end{array}
$$

Proof. To prove this equality, we need to display a map between the underlying vector spaces which respects the frames.

Let $A^{\prime}$ be the boundary of $\left(\overline{\mathcal{M}_{0, n+3}} \backslash A_{\mathbf{k}}\right) \times\left(\overline{\mathcal{M}_{0, m+3}} \backslash A_{\mathbf{l}}\right)$, it is equal to the divisor of singularities of $\omega_{\mathbf{k}} \wedge \omega_{\mathbf{l}}$ on $\overline{\mathcal{M}_{0, n+3}} \times \overline{\mathcal{M}_{0, m+3}}$. 
Let $A_{0}=\beta^{-1}\left(A^{\prime}\right)$ and let $B_{0}$ be the Zariski closure of the boundary of $\Phi_{0}=$ $\beta^{-1}\left(\Phi_{n} \times \Phi_{m}\right)$. Let $B_{n, m}$ be the Zariski closure of the boundary of $\Phi_{n} \times \Phi_{m}$. The map $\beta$ induces a map:

$$
\begin{aligned}
& \left(\overline{\mathcal{M}_{0, n+m+3}} \backslash A_{0} ; B_{0}^{A_{0}}\right)^{\beta} \longrightarrow\left(\left(\overline{\mathcal{M}_{0, n+3}} \backslash A_{\mathbf{k}}\right) \times\left(\overline{\mathcal{M}_{0, m+3}} \backslash A_{\mathbf{l}}\right) ; \beta\left(B_{0}\right)^{A^{\prime}}\right) \\
& \left(\left(\overline{\mathcal{M}_{0, n+3}} \backslash A_{\mathbf{k}}\right) \times\left(\overline{\mathcal{M}_{0, m+3}} \backslash A_{\mathbf{l}}\right) ; B_{n, m}^{A^{\prime}}\right) .
\end{aligned}
$$

We introduce the vertical inclusion $\alpha$ because $B_{0}$ does not map onto $B_{n, m}$ via $\beta$. The map $\alpha$ induces a map on the mixed Tate motives:

$$
\begin{aligned}
\mathrm{H}^{n+m} & \left.\left(\overline{\mathcal{M}_{0, n+3}} \backslash A_{\mathbf{k}}\right) \times\left(\overline{\mathcal{M}_{0, m+3}} \backslash A_{\mathbf{l}}\right) ; \beta\left(B_{0}\right)^{A^{\prime}}\right) \stackrel{\alpha^{*}}{\longrightarrow} \\
& \mathrm{H}^{n+m}\left(\left(\overline{\mathcal{M}_{0, n+3}} \backslash A_{\mathbf{k}}\right) \times\left(\overline{\mathcal{M}_{0, m+3}} \backslash A_{\mathbf{l}}\right) ; B_{n, m}^{A^{\prime}}\right) .
\end{aligned}
$$

The frames on the RHS of (14) are given by $\left[\Phi_{n} \times \Phi_{m}\right]$ and $\left[\omega_{\mathbf{k}} \wedge \omega_{\mathbf{l}}\right]$. Applying lemma 3.3 to $(14),\left[\Phi_{n} \times \Phi_{m}\right]$ induces a map $\tilde{\Phi}$ from $\mathbb{Q}(0)$ to the $-2(n+m)$ graded part of the LHS of (14). In fact, since $\alpha$ is the identity map, we have $[\tilde{\Phi}]=\left[\Phi_{n} \times \Phi_{m}\right]$, so $\left[\Phi_{n} \times \Phi_{m}\right]$ and $\left[\omega_{\mathbf{k}} \wedge \omega_{\mathbf{l}}\right]$ give frames on the LHS of (14) which are compatible with the map $\alpha^{*}$.

The map $\beta$ induces a map on the mixed Tate motives:

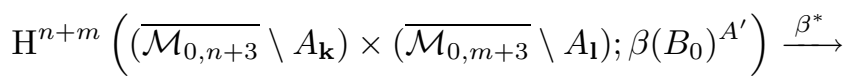

$$
\begin{aligned}
& \mathrm{H}^{n+m}\left(\overline{\mathcal{M}_{0, n+m+3}} \backslash A_{0} ; B_{0}^{A_{0}}\right) .
\end{aligned}
$$

On the RHS of (15) the frames are given by $\left[\omega_{0}\right]$ where $\omega_{0}$ is $\beta^{*}\left(\omega_{\mathbf{k}} \wedge \omega_{\mathbf{l}}\right)$ and $\left[\Phi_{0}\right]=\left[\beta^{-1}\left(\Phi_{n} \times \Phi_{m}\right)\right]$, which are compatible with the map $\beta^{*}$.

Now we can prove the proposition. The Künneth formula gives a map:

$$
\begin{aligned}
\mathrm{H}^{n}\left(\overline{\mathcal{M}_{0, n+3}} \backslash A_{\mathbf{k}} ; B_{n}^{A_{\mathbf{k}}}\right) \otimes \mathrm{H}^{m}\left(\overline{\mathcal{M}_{0, m+3}} \backslash A_{\mathbf{l}} ; B_{m}^{A_{1}}\right) & \longrightarrow \\
& \left.\mathrm{H}^{n+m}\left(\overline{\left(\mathcal{M}_{0, n+3}\right.} \backslash A_{\mathbf{k}}\right) \times\left(\overline{\mathcal{M}_{0, m+3}} \backslash A_{\mathbf{l}}\right) ; B_{n, m}^{A^{\prime}}\right) .
\end{aligned}
$$

By theorem 3.1, this map also respects the frames, so the associated framed mixed Tate motives are equal. By (14),

$$
\left.\left[\mathrm{H}^{n+m}\left(\overline{\left(\mathcal{M}_{0, n+3}\right.} \backslash A_{\mathbf{k}}\right) \times\left(\overline{\mathcal{M}_{0, m+3}} \backslash A_{\mathbf{l}}\right) ; B_{n, m}^{A^{\prime}}\right) ;\left[\omega_{\mathbf{k}} \otimes \omega_{\mathbf{l}}\right] ;\left[\Phi_{n} \times \Phi_{m}\right]\right]
$$

is equal to

$$
\left[\mathrm{H}^{n+m}\left(\left(\overline{\mathcal{M}_{0, n+3}} \backslash A_{\mathbf{k}}\right) \times\left(\overline{\mathcal{M}_{0, m+3}} \backslash A_{\mathbf{l}}\right) ; \beta\left(B_{0}\right)^{A^{\prime}}\right) ;\left[\omega_{\mathbf{k}} \otimes \omega_{\mathbf{l}}\right] ;\left[\Phi_{n} \times \Phi_{m}\right]\right],
$$

which, using (15), is equal to

$$
\left[\mathrm{H}^{n+m}\left(\overline{\mathcal{M}_{0, n+m+3}} \backslash A_{0} ; B_{0}^{A_{0}}\right) ;\left[\omega_{0}\right] ;\left[\Phi_{0}\right]\right] .
$$

It remains to show that

$$
\begin{aligned}
& {\left[\mathrm{H}^{n+m}\left(\overline{\mathcal{M}_{0, n+m+3}} \backslash A_{0} ; B_{0}^{A_{0}}\right) ;\left[\omega_{0}\right] ;\left[\Phi_{0}\right]\right]=} \\
& \qquad \sum_{\sigma}\left[\mathrm{H}^{n+m}\left(\overline{\mathcal{M}_{0, n+m+3}} \backslash A_{\sigma} ; B_{n+m}^{A_{\sigma}}\right) ;\left[\omega_{\sigma}\right] ;\left[\Phi_{n+m}\right]\right] .
\end{aligned}
$$

In the LHS of (16), $B_{0}$ being included in $B_{\mathrm{sh}}=\bigcup_{\sigma} B_{\sigma}$, we can replace $B_{0}$ by $B_{\text {sh }}$ using lemma 3.6 . 
As $\left[\Phi_{0}\right]=\sum_{\sigma}\left[\Phi_{n+m}^{\sigma}\right]$, lemma 3.2 shows that the LHS of 16 is equal to

$$
\sum_{\sigma}\left[\mathrm{H}^{n+m}\left(\overline{\mathcal{M}_{0, n+m+3}} \backslash A_{0} ; B_{\mathrm{sh}}^{A_{0}}\right) ;\left[\omega_{0}\right] ;\left[\Phi_{n+m}^{\sigma}\right]\right] .
$$

Using the fact that $B_{\sigma} \subset B_{\mathrm{sh}}$ and an inclusion map, lemma 3.6 shows that this framed motive is equal to

$$
\sum_{\sigma}\left[\mathrm{H}^{n+m}\left(\overline{\mathcal{M}_{0, n+m+3}} \backslash A_{0} ; B_{\sigma}^{A_{0}}\right) ;\left[\omega_{0}\right] ;\left[\Phi_{n+m}^{\sigma}\right]\right] .
$$

As the divisor of singularities $A$ of $\omega_{0}$ is included in $A_{0}$, using lemma 3.6 we can replace $A_{0}$ by $A$ in this framed motive. Then permuting the points gives an equality of framed motives on each term of the sum,

$$
\left[\mathrm{H}^{n+m}\left(\overline{\mathcal{M}_{0, n+m+3}} \backslash A_{0} ; B_{\sigma}^{A_{0}}\right) ;\left[\omega_{0}\right] ;\left[\Phi_{n+m}^{\sigma}\right]\right],
$$

with

$$
\left[\mathrm{H}^{n+m}\left(\overline{\mathcal{M}_{0, n+m+3}} \backslash A_{\sigma} ; B_{n+m}^{A_{\sigma}}\right) ;\left[\omega_{\sigma}\right] ;\left[\Phi_{n+m}\right]\right] .
$$

Thus, we obtain the desired formula:

$$
\begin{array}{r}
{\left[\mathrm{H}^{n}\left(\overline{\mathcal{M}_{0, n+3}} \backslash A_{\mathbf{k}} ; B_{n}^{A_{\mathbf{k}}}\right) ;\left[\omega_{\mathbf{k}}\right] ;\left[\Phi_{n}\right]\right] \cdot\left[\mathrm{H}^{m}\left(\overline{\mathcal{M}_{0, m+3}} \backslash A_{\mathbf{l}} ; B_{m}^{A_{\mathbf{l}}}\right) ;\left[\omega_{\mathbf{l}}\right] ;\left[\Phi_{m}\right]\right]=} \\
\sum_{\sigma \in \operatorname{sh}((1, \ldots, n),(n+1, \ldots, n+m))}\left[\mathrm { H } ^ { n + m } \left(\overline{\left.\left.\mathcal{M}_{0, n+m+3} \backslash A_{\sigma} ; B_{n+m}^{A_{\sigma}}\right) ;\left[\omega_{\sigma}\right] ;\left[\Phi_{n+m}\right]\right] .}\right.\right.
\end{array}
$$

\section{The STUfFle CASE}

The goal of this section is to be able to translate all the calculations done in Section 1.3 into a motivic context. In order to achieve this goal, we need to define, for all $n$ greater than 2 , a variety $X_{n} \rightarrow \mathbb{A}^{n}$ resulting from successive blow-ups of $\mathbb{A}^{n}$ together with a differential form $\Omega_{k_{1}, \ldots, k_{p}}^{s}$ for any tuple of integer $\left(k_{1}, \ldots, k_{p}\right)$ (with $k_{1}+\cdots k_{p}=n$ ) and any permutation $s$ of $\llbracket 1, n \rrbracket$. We use the $X_{n}$ to give another definition of the motivic multiple zeta value which we show is actually equal to Goncharov-Manin's. Then, using a natural map from $X_{n+m}$ to an open subset of $\mathcal{M}_{0, n+m+3}$, we use this new definition to prove that the motivic multiple zeta values satisfy the stuffle relation.

\subsection{Blow-up preliminaries.}

Lemma 4.1 (Flag Blow-up Lemma; [Uly02].). Let $V_{0}^{1} \subset V_{0}^{2} \subset \cdots V_{0}^{r} \subset W_{0}$ be a flag of smooth subvarieties in a smooth algebraic variety $W_{0}$. For $k=1, \ldots, r$, define inductively $W_{k}$ as the blow-up of $W_{k-1}$ along $V_{k-1}^{k}$, then $V_{k}^{k}$ as the exceptional divisor in $W_{k}$ and $V_{k}^{i}, k \leqslant i$, as the proper transform of $V_{k-1}^{i}$ in $W_{k}$. Then the preimage of $V_{0}^{r}$ in the resulting variety $W_{r}$ is a normal crossing divisor $V_{r}^{1} \cup \cdots \cup V_{r}^{r}$.

If $\mathscr{F}$ is a flag of subvarieties $V_{0}^{i}$ in a smooth algebraic variety $W_{0}$ as in the previous lemma, the resulting space $W_{s}$ will be denoted by $\mathrm{Bl}_{\mathscr{F}} W_{0}$.

Theorem 4.2 ([Hu03]). Let $X_{0}$ be an open subset of a nonsingular algebraic variety $X$. Assume that $X \backslash X_{0}$ can be decomposed as a finite union $\cup_{i \in I} D_{i}$ of closed irreducible subvarieties such that

(1) for all $i \in I, D_{i}$ is smooth;

(2) for all $i, j \in I, D_{i}$ and $D_{j}$ meet cleanly, that is the scheme-theoretic intersection is smooth and the intersection of the tangent space $T_{X}\left(D_{i}\right) \cap T_{X}\left(D_{j}\right)$ is the tangent space of the intersection $T_{X}\left(D_{i} \cap D_{j}\right)$;

(3) for all $i, j \in I, D_{i} \cap D_{j}=\emptyset$; or a disjoint union of $D_{l}$. 
The set $\mathcal{D}=\left\{D_{i}\right\}_{i \in I}$ is then a poset. Let $k$ be the rank of $\mathcal{D}$. Then there is a sequence of well-defined blow-ups

$$
\mathrm{Bl}_{\mathcal{D}} X \rightarrow \mathrm{Bl}_{\mathcal{D} \leqslant k-1} X \rightarrow \cdots \rightarrow \mathrm{Bl}_{\mathcal{D} \leqslant 0} X \rightarrow X
$$

where $\mathrm{Bl}_{\mathcal{D} \leqslant 0} X \rightarrow X$ is the blow-up of $X$ along $D_{i}$ of rank 0 , and, inductively, $\mathrm{Bl}_{\mathcal{D} \leqslant r} X \rightarrow \mathrm{Bl}_{\mathcal{D} \leqslant r-1} X$ is the blow-up of $\mathrm{Bl}_{\mathcal{D} \leqslant r-1} X$ along the proper transforms of $D_{j}$ of rank $r$, such that

(1) $\mathrm{Bl}_{\mathcal{D}} X$ is smooth;

(2) $\mathrm{Bl}_{\mathcal{D}} X \backslash X_{0}=\bigcup_{i \in I} \widetilde{D_{i}}$ is a divisor with normal crossings;

(3) for any integer $k, \widetilde{D_{i_{1}}} \cap \cdots \cap \widetilde{D_{i_{k}}}$ is non-empty if and only if, up to numbering, $D_{i_{1}} \subset \cdots \subset D_{i_{k}}$ form a chain in the poset $\mathcal{D}$. Consequently, $\widetilde{D_{i}}$ and $\widetilde{D_{j}}$ meet if and only if $D_{i}$ and $D_{j}$ are comparable. lary.

The fact that blow-ups are local constructions yields directly the following corol-

Corollary 4.3 (Flags blow-up sequence). Let $X$ and $\mathcal{D}$ be as in the previous theorem. Let $\mathscr{F}_{1}, \ldots, \mathscr{F}_{k}$ be flags of subvarieties of $\mathcal{D}$ such that

(1) $\mathscr{F}_{1}, \ldots, \mathscr{F}_{k}$ is a partition of $\mathcal{D}$,

(2) if $D$ is in some $\mathscr{F}_{i}$, then for all $D^{\prime} \in \mathcal{D}$ with $D^{\prime}<D$ there exists some $j \leqslant i$ such that $D^{\prime} \in \mathscr{F}_{j}$.

If $\mathscr{F}_{j}^{i}$ denotes the flag of the proper transform of elements of $\mathscr{F}_{j}^{i-1}$ in

$$
\mathrm{Bl}_{\mathscr{F}_{i}^{i-1}}\left(\cdots\left(\mathrm{Bl}_{\mathscr{F}_{1}} X\right) \cdots\right) \text {, }
$$

then

$$
\mathrm{Bl}_{\mathcal{D}} X=\mathrm{Bl}_{\mathscr{F}_{k}^{k-1}}\left(\cdots\left(\mathrm{Bl}_{\mathscr{F}_{1}} X\right) \cdots\right) .
$$

We will denote such a sequence of blow-ups by

$$
\mathrm{Bl}_{\mathcal{F}_{k}, \ldots, \mathcal{F}_{1}} X \text {. }
$$

As we want to apply these results in order to have a motivic description of the stuffle product in terms of blow-ups, we need some more precise information about what sort of motives arise from the construction of Theorem 4.2. Following the notation of the article [Hu03], in particular using the proof of theorems 1.4, 1.7 and Corollary 1.6, we deduce the following proposition:

Proposition 4.4. Suppose that $X$ and $\mathcal{D}=\cup D_{i}$ as in proposition 4.2 are such that $X$ and all the $D_{i}$ are Tate varieties. Let $\mathcal{E}^{r+1}$ be the set of exceptional divisors of $\mathrm{Bl}_{\mathcal{D} \leqslant r} X \rightarrow X$. Then all possible intersections of strata of $\mathcal{D}^{r+1} \cup \mathcal{E}^{r+1}$ are Tate Varieties and so is $\mathrm{Bl}_{\mathcal{D} \leqslant r} X$.

Proof. Mainly following the proof of theorem 1.7 in [Hu03], we use an induction on $r$.

If $r=0$ then $\mathrm{Bl}_{\mathcal{D} \leqslant 0} X \rightarrow X$ is the blow-up along the disjoint subvarieties $D_{i}$ of rank 0 .

All the exceptional divisors in $\mathcal{E}^{1}$ are of the form $\mathbb{P}\left(N_{X} D_{i}\right)$ (with $D_{i}$ of rank 0 ), and as the $D_{i}$ are Tate, so are the exceptional divisors.

The blow-up formula

$$
h\left(X_{Z}\right)=\mathfrak{H}(X) \bigoplus_{i=0}^{d-1} h(Z)(-i)[-2 i]
$$

tell us that the blow-up of a Tate variety $X$ along some Tate variety $Z$ of codimension $d$ is a Tate variety. Then $\mathrm{Bl}_{\mathcal{D} \leqslant 0} X$ is Tate. Moreover, if $D_{i}^{1}$ is an element of $\mathcal{D}^{1}$, then it is the proper transform of an element $D_{i}$ in $\mathcal{D}$ of rank greater than 1. 
Theorem 1.4 in [Hu03] tells us that $D_{i}^{1}=\mathrm{Bl}_{D_{j} \subset D_{i} ; \operatorname{rank}\left(D_{j}\right)=0} D_{j}$ and is therefore a Tate variety.

We now need to show that all intersections of strata of $\mathcal{D}^{1} \cap \mathcal{E}^{1}$ are Tate. As $\mathrm{Bl}_{\mathcal{D} \leqslant 0} X \rightarrow X$ is a blow-up along disjoint subvarieties, the exceptional divisors are disjoint and we conclude that elements in $\mathcal{E}^{1}$ do not intersect.

Let $D_{i}^{1}$ and $D_{j}^{1}$ be two elements of $\mathcal{D}^{1}$; they are the proper transforms of $D_{i}$ and $D_{j}$ in $\mathcal{D}$. If $D_{i} \cap D_{j}=\emptyset$ then, the same hold for their proper transforms and there is nothing to prove. Otherwise, by assumption, $D_{i} \cap D_{j}$ is a disjoint union $\cup D_{l}$. If the maximal rank of the $D_{l}$ is 0 then Lemma 2.1 in [Hu03] ensures that the proper transforms have an empty intersection. If the maximal rank of the $D_{l}$ is greater than 1 the fact that $D_{i}$ and $D_{j}$ meet cleanly ensures that the proper transform of the intersection is the intersection of the proper transforms, that is

$$
D_{i}^{1} \cap D_{j}^{1}=\mathrm{Bl}_{D_{l} \subset D_{i} \cap D_{j} ; \operatorname{rank}\left(D_{l}\right)=0} D_{i} \cap D_{j}
$$

and the intersection is Tate because $D_{i} \cap D_{j}$ is a disjoint union of $D_{l}$ which are Tate. Moreover from theorem 1.4 ([Hu03]) we have $D_{i}^{1} \cap D_{j}^{1}=\cup D_{l}^{1}$. Thus we can consider only intersections of the form $E^{1} \cap D_{i}^{1}$ with $E^{1}$ in $\mathcal{E}^{1}$ and $D_{i}^{1}$ in $\mathcal{D}^{1}$. Such an intersection is non empty if and only if $E^{1}$ comes from an element $D_{j}$ of rank 0 in $\mathcal{D}$ with $D_{j} \subset D_{i}$. Then $E^{1} \cap D_{i}^{1}$ is $\mathbb{P}\left(N_{D_{i}} D_{j}\right)$ and is a Tate variety.

Assume the statement is true for $\mathrm{Bl}_{\mathcal{D} \leqslant r-1} X, \mathcal{E}^{r}$ and $\mathcal{D}^{r}$. By corollary 1.6 in [Hu03], the blow-up $\mathrm{Bl}_{\mathcal{D} \leqslant r} X \rightarrow \mathrm{Bl}_{\mathcal{D} \leqslant r-1} X$ is

$$
\mathrm{Bl}_{\mathcal{D}_{\leqslant 0}^{r}}\left(\mathrm{Bl}_{\mathcal{D} \leqslant r} X\right) \longrightarrow \mathrm{Bl}_{\mathcal{D} \leqslant r-1} X \text {. }
$$

This is a blow-up along elements in $\mathcal{D}^{r}$ of rank $r$ which by assumption are Tate, as $\mathrm{Bl}_{\mathcal{D} \leqslant r-1} X$. Then, $\mathrm{Bl}_{\mathcal{D} \leqslant r} X$ and the new exceptional divisors are Tate. The other exceptional divisors are proper transforms of elements in $\mathcal{E}^{r}$ and are of the form

$$
E_{i}^{r+1}=\mathrm{Bl}_{E_{i}^{r} \cap D_{l}^{r} ; \operatorname{rank}\left(D_{l}\right)=r} E_{i}^{r}
$$

with $E_{i}^{r}$ in $\mathcal{E}^{r}$ and $D_{l}^{r}$ in $\mathcal{D}^{r}$ coming from some $D_{l}$ in $\mathcal{D}$. As by the induction hypothesis both $E_{i}^{r}$ and $E_{i}^{r} \cap D_{l}^{r}$ are Tate, $E_{i}^{r+1}$ is a Tate variety. The same argument proves that all elements in $\mathcal{D}^{r+1}$ are Tate. As previously the intersection of two elements in $\mathcal{D}^{r+1}$ is either empty or the proper transform of the intersection of two elements in $\mathcal{D}^{r}$, again this proper transform is Tate.

Theorem 1.4 tells us that the intersection $D_{i}^{r+1} \cap D_{j}^{r+1}$ of two elements of $\mathcal{D}^{r+1}$ is either empty or the union of some elements $D_{l}^{r+1}$ in $\mathcal{D}^{r+1}$. Then, to prove that all possible intersections of strata of $\mathcal{E}^{r+1} \cup \mathcal{D}^{r+1}$ is Tate, it is enough to prove that the intersection of some $D_{i}^{r+1}$ with any intersection $E_{1}^{r+1} \cap \cdots E_{k}^{r+1}$ is Tate.

If two of the $E_{i}^{r+1}$ are exceptional divisors of $\mathrm{Bl}_{\mathcal{D}_{\leqslant 0}^{r}}\left(\mathrm{Bl}_{\mathcal{D} \leqslant r} X\right) \rightarrow \mathrm{Bl}_{\mathcal{D} \leqslant r-1} X$ then the intersection is empty because the corresponding strata $D_{i}^{r}$ and $D_{j}^{r}$ have an empty intersection (they have been separated at a previous stage).

Hence at most one of the $E_{i}^{r+1}$ is an exceptional divisor coming from the last blow-up, and we can suppose that the strata $D_{i}^{r+1}, E_{1}^{r+1}, \ldots, E_{k-1}^{r+1}$ come from strata at the previous stage $D_{i}^{r}, E_{1}^{r}, \ldots, E_{k-1}^{r}$.

- Suppose that $E_{k}^{r+1}$ is the proper transform of an exceptional divisor $E_{k}^{r}$ in $\mathcal{E}^{r}$. The subvariety $Y=D_{i}^{r} \cap E_{1}^{r} \cap \cdots E_{k}^{r}$ is Tate by the induction hypothesis and its proper transform is

$$
\mathrm{Bl}_{D_{j}^{r} \cap Y ; \operatorname{rank}\left(D_{j}\right)=r} Y
$$

which is a Tate variety $\left(D_{j}^{r} \cap Y\right.$ is either empty or Tate and $Y$ is Tate). On the other side the proper transform of $Y$ is the intersection $D_{i}^{r+1} \cap E_{1}^{r+1} \cap$ $\cdots \cap E_{k}^{r+1}$, which is therefore Tate. 
- Suppose that $E_{k}^{r}$ is the exceptional divisor coming from the blow-up of $\mathrm{Bl}_{\mathcal{D} \leqslant r-1} X$ along $D_{j}^{r}$. Let $Y$ be the intersection $D_{i}^{r} \cap E_{1}^{r} \cap \cdots E_{k-1}^{r}$. Then $D_{j}^{r} \cap Y$ is either empty or a Tate variety In the first case the intersection $D_{i}^{r+1} \cap E_{1}^{r+1} \cap \cdots \cap E_{k}^{r+1}$ is empty. In the latter case we have

$$
D_{i}^{r+1} \cap E_{1}^{r+1} \cap \cdots \cap E_{k}^{r+1}=\mathbb{P}\left(N_{Y} Y \cap D_{j}^{r}\right)
$$

which is Tate.

4.2. The space $X_{n}$ and some of its properties. Let $n$ be an integer greater than 2 and let $x_{1}, \ldots, x_{n}$ be the natural coordinates on $\mathbb{A}^{n}$. We define the divisors $A_{I}, B_{i}^{0}, B_{i}^{1}, A_{n}, B_{n}, D_{n}^{1}, D_{n}^{0}$ and $D_{n}$ as follows:

- for all non empty subsets $I$ of $\llbracket 1, n \rrbracket, A_{I}$ is the divisor defined by

$$
1-\prod_{i \in I} x_{i}=0
$$

- for all $i \in \llbracket 1, n \rrbracket, B_{i}^{0}$ is the divisor defined by $x_{i}=0$;

- for all $i \in \llbracket 1, n \rrbracket, B_{i}^{1}=A_{\{i\}}$ is the divisor defined by $1-x_{i}=0$;

- $B_{n}$ is the union $\left(\bigcup_{i} B_{i}^{0}\right) \bigcup\left(\bigcup_{i} B_{i}^{1}\right)$;

- $A_{n}$ is the union $\bigcup_{I \subset \llbracket 1, n \rrbracket ; I I \geqslant 2} A_{I}$;

- $D_{n}^{1}$ is the union $\bigcup_{I \subset \llbracket 1, n \rrbracket ; I \neq \emptyset} A_{I}$;

- $D_{n}^{0}$ is the union $\bigcup_{i} B_{i}^{0}$;

- $D_{n}$ is the union $D_{n}^{0} \cup D_{n}^{1}$.

Remark 4.5. The divisor $B_{n}$ is the Zariski closure of the boundary of the real cube $C_{n}=[0,1]^{n}$ in $\mathbb{A}^{n}(\mathbb{R})$.

As the divisor $D_{n}$ is not normal crossing, we would like to find a suitable succession of blow-ups that will allow us to have a normal crossing divisor $\widehat{D}_{n}$ over $D_{n}$. In order to achieve this we first need the following remark and lemmas.

Remark 4.6. Let $I$ be a non-empty subset of $\llbracket 1, n \rrbracket$ and $x=\left(x_{1}, \ldots, x_{n}\right)$ a point in $A_{I}$. Then the normal vector of $A_{I}$ at the point $x$ is

$$
n_{\mid x}^{A_{I}}=\sum_{i \in I} \frac{1}{x_{i}} \mathrm{~d} x_{i} .
$$

Therefore, if $I$ and $J$ are two distinct non-empty subsets of $\llbracket 1, n \rrbracket$, the intersection of $A_{I}$ and $A_{J}$ is transverse.

Lemma 4.7. Let $I_{1}, \ldots, I_{k}$ be $k$ subsets of $\llbracket 1, n \rrbracket$ and $X$ the intersection $A_{I_{1}} \cap \cdots \cap$ $A_{I_{k}} \subset \mathbb{A}^{n}$. Then, there exist non negative integers $r$ and $s$ with $r>0, s+r \leqslant n$ and integers $c_{1}, \ldots, c_{r}$ such that $X$ is isomorphic to

$$
\mathbb{A}^{s} \times \mathbb{G}_{m}^{n-s-r} \times \prod_{i=1}^{r}\left\{x^{c_{i}}=1\right\} .
$$

Proof. If $\left|I_{1} \cup \cdots \cup I_{k}\right|=a<n$ then $X$ is isomorphic to $\left(A_{I_{1}}^{\prime} \cap \cdots \cap A_{I_{k}}^{\prime}\right) \times \mathbb{A}^{n-a} \subset$ $\mathbb{A}^{a} \times \mathbb{A}^{n-a}$, where the $A_{I_{i}}^{\prime}$ are defined by the same equations, $1-\prod_{j \in I_{i}} x_{j}=0$, that define $A_{I_{i}}$ but viewed in $\mathbb{A}^{a}$ instead of $\mathbb{A}^{n}$. Putting $s=n-a$, it is enough to show that we have

$$
X^{\prime}=\left(A_{I_{1}}^{\prime} \cap \cdots \cap A_{I_{k}}^{\prime}\right) \simeq \mathbb{G}_{m}^{a-r} \times \prod_{i=1}^{r}\left\{x^{c_{i}}=1\right\} .
$$

We now assume that $\left|I_{1} \cup \cdots \cup I_{r}\right|=n$. 
For any tuple $\lambda=\left(\lambda_{1}, \ldots, \lambda_{n}\right)$ of integers and any $x$ in $\mathbb{A}^{n}$, let $x^{\lambda}$ denote the product $\prod_{j=1}^{n} x_{j}^{\lambda_{j}}$. For $i$ in $\llbracket 1, n \rrbracket$, let $a_{i}=\left(a_{i 1}, \ldots, a_{i n}\right)$ be the element of $\mathbb{Z}^{n}$ defined by

$$
\forall j, 1 \leqslant j \leqslant n \quad a_{i j}=\delta_{I_{i}}(j)
$$

where $\delta_{I_{i}}$ is the characteristic function of the set $I_{i}$. Using these notations, $X$ is defined by the equations

$$
x^{a_{1}}=\cdots=x^{a_{k}}=1 .
$$

Let $L$ be the submodule of $\mathbb{Z}^{n}$ spanned by $a_{1}, \ldots, a_{n}$, and let $r, r \leqslant k$, be its rank. For $\lambda$ in $L$, writing

$$
\lambda=\alpha_{1} a_{1}+\cdots \alpha_{k} a_{k},
$$

we see that for any $x$ in $\mathbb{A}^{n}$ we have

$$
x^{\lambda}=\prod_{i=1}^{n}\left(x^{a_{i}}\right)^{\alpha_{i}} .
$$

In consequence, $x$ is in $X$ if and only if for all $\lambda$ in $L$ one has $x^{\lambda}=1$.

The module $L$ being a submodule of the free $\mathbb{Z}$-module $\mathbb{Z}^{n}$, we have a basis $f_{1}, \ldots, f_{n}$ of $\mathbb{Z}^{n}$ and integers $c_{1}, \ldots, c_{r}$ such that

$$
L=f_{1} \cdot c_{1} \mathbb{Z} \oplus \cdots \oplus f_{r} \cdot c_{r} \mathbb{Z} .
$$

As an element $x$ of $\mathbb{A}^{n}$ is in $X$ if and only if

$$
\forall \lambda \in L, x^{\lambda}=1
$$

we deduce that $x$ is in $X$ if and only if

$$
\left(x^{f_{1}}\right)^{c_{1}}=1, \quad \ldots, \quad\left(x^{f_{r}}\right)^{c_{r}}=1
$$

and $X$ is defined by the above equation.

Let $e_{1}, \ldots, e_{n}$ be the canonical basis of $\mathbb{Z}^{n}$ and $\varphi$ be the isomorphism of $\mathbb{Z}^{n}$ sending each $f_{i}$ to $e_{i}$ for $i$ in $\llbracket 1, n \rrbracket$. Let $(\varphi i j)_{\substack{1 \leqslant i \leqslant n \\ 1 \leqslant i \leqslant n}}$ denote the matrix of $\varphi$ in the canonical basis. The morphism $\varphi$ induces a morphism $\tilde{\varphi}$ from $\mathbb{G}_{m}^{n}$ to $\mathbb{G}_{m}^{n}$ defined on the coordinates by

$$
\tilde{\varphi}\left(x_{j}\right)=\prod_{i=1}^{n} x_{i}^{\varphi_{i j}},
$$

such that $\tilde{\varphi}$ sends $X$ to the subvariety $\tilde{X}$ defined by

$$
x^{\varphi\left(c_{1} f_{1}\right)}=1, \quad \ldots, \quad x^{\varphi\left(c_{r} f_{r}\right)}=1 .
$$

As $\varphi\left(c_{i} f_{i}\right)=c_{i} e_{i}$ for all $i$ in $\llbracket 1, n \rrbracket, \tilde{X}$ is in fact defined by

$$
x_{1}^{c_{1}}=1, \quad \cdots, \quad x_{r}^{c_{r}}=1 .
$$

The morphism $\varphi$ being invertible, $\tilde{\varphi}$ is an isomorphism and $X$ is isomorphic to

$$
\mathbb{G}_{m}^{n-r} \times \prod_{i=1}^{r}\left\{x^{c_{i}}=1\right\} .
$$

Lemma 4.8. Let $I_{1}, \ldots, I_{k}$ be $k$ subsets of $\llbracket 1, n \rrbracket$ and $X$ the intersection $A_{I_{1}} \cap$ $\cdots \cap A_{I_{r}} \subset \mathbb{A}^{n}$. Then, the normal bundle $N_{\mathbb{A}^{n}} X$ is spanned by the normal bundle $N_{\mathbb{A}^{n}} A_{I_{1}}, \ldots, N_{\mathbb{A}^{n}} A_{I_{k}}$. 
Proof. As in the proof of the previous lemma, it is enough to suppose that $\mid I_{1} \cup$ $\cdots \cup I_{k} \mid=n$.

Each of the $N_{\mathbb{A}^{n}} A_{I_{i}}$ is a subbundle of $N_{\mathbb{A}^{n}} X$. Thus, as $X$ is smooth, checking that we have the equality of dimensions is enough. Using equation (18), we see that at a point $x$ of $X$ the dimension of the vector space $\operatorname{Vect}\left(\mathrm{n}_{\mid \mathrm{x}}^{\mathrm{A}_{\mathrm{I}_{1}}}, \ldots, \mathrm{n}_{\mid \mathrm{x}} \mathrm{A}_{\mathrm{I}_{\mathrm{k}}}\right)$ is equal to the rank of the matrix

$$
M=\left(\frac{1}{x_{j}} \delta_{I_{i}}(j)\right)_{\substack{1 \leqslant i \leqslant k \\ 1 \leqslant j \leqslant n}}
$$

where $\delta_{I_{i}}(j)$ is the characteristic function of the set $I_{i}$. The rank of $M$ is that of the matrix $\left(\delta_{I_{i}}(j)\right)_{\substack{1 \leqslant i \leqslant k \\ 1 \leqslant j \leqslant n}}$, that is the rank of the $\mathbb{Z}$-module $L$ spanned by $a_{1}, \ldots, a_{k}$ defined in the previous proof. By the proof of Lemma 4.7, the rank of $L$ is the codimension of $L$, which completes the proof of lemma 4.8 .

Lemma 4.9. Let $\mathcal{D}_{n}^{1}$ be the poset (for the inclusion) formed by all the irreducible components of all possible intersections of divisors $A_{I}$. Then the poset $\mathcal{D}_{n}^{1}$ satisfies the conditions (1), (2) and (3) of theorem 4.2.

Proof. The intersection condition (3) follows from the definition of $\mathcal{D}_{n}^{1}$. From Lemma 4.7, any possible intersection $X=A_{I_{1}} \cap \cdots \cap A_{I_{k}}$ is isomorphic to $\mathbb{A}^{s} \times$ $\mathbb{G}_{m}^{n-s-r} \times \prod_{i=1}^{r}\left\{x^{c_{i}}=1\right\}$ for some non negative integers $r$ and $s$ and integers $c_{i}$, thus $X$ is smooth and its irreducible components are all smooth.

Let $S_{1}$ and $S_{2}$ be two elements of $\mathcal{D}_{n}^{1}$. To show that $S_{1}$ and $S_{2}$ meet cleanly, it is enough to show that the normal bundle of the intersection is spanned by the normal bundles of $S_{1}$ and $S_{2}$, that is

$$
N_{\mathbb{A}^{n}}\left(S_{1} \cap S_{2}\right)=N_{\mathbb{A}^{n}}\left(S_{1}\right)+N_{\mathbb{A}^{n}}\left(S_{2}\right)
$$

As $S_{1}$ and $S_{2}$ are intersections of some $A_{I}$, it is enough to show that the normal bundle of $A_{I_{1}} \cap \cdots \cap A_{I_{k}}$ is spanned by the normal vectors of the $A_{I_{j}}$ and that is ensured by lemma 4.8 .

Applying the construction of theorem 4.2 with $\mathcal{D}=\mathcal{D}_{n}^{1}$ and $X=\mathbb{A}^{n}$ leads to a variety $X_{n} \stackrel{p_{n}}{\rightarrow} \mathbb{A}^{n}$, which results from successive blow-ups of all the strata of $\mathcal{D}_{n}^{1}$ such that the preimage $\widehat{\mathcal{D}_{n}^{1}}$ of $\mathcal{D}_{n}^{1}$ is a normal crossing divisor. We will write $\widehat{D_{n}^{1}}$ to denote the preimage of $D_{n}^{1}$.

Lemma 4.10. Let $\widehat{D_{n}^{0}}$ be the proper transform in $X_{n}$ of the divisor $D_{n}^{0}$. Then $\widehat{D}_{n}=\widehat{D_{n}^{1}} \cup \widehat{D_{n}^{0}}$ is a normal crossing divisor.

Proof. Let $I$ be a non-empty subset of $\llbracket 1, n \rrbracket, \widehat{B_{I}^{0}}$ (resp. $B_{I}^{0}$ ) be the intersection in $X_{n}$ (resp. $\mathbb{A}^{n}$ ) of divisors $\left\{x_{i}=0\right\}$ for $i$ in $I$. And let $\widehat{S_{1}}, \ldots, \widehat{S_{k}}$ be strata of $\widehat{\mathcal{D}_{1}^{n}}$ such that the intersection of the $\widehat{S}_{i}$ is non-empty. We want to show that there is a neighbourhood $V$ of $\widehat{B_{I}^{0}} \cap \widehat{S_{1}} \cap \cdots \cap \widehat{S_{k}}$ such that $V \cap \widehat{D}_{n}$ is normal crossing. By theorem 4.2 , the $\widehat{S}_{i}$ come from strata of $\mathcal{D}_{n}^{1}, S_{1} \subset \cdots \subset S_{k}$. As the intersection of the $\widehat{S}_{i}$ 's with $\widehat{B_{I}^{0}}$ is non-empty, the intersection of $B_{I}^{0}$ with $S_{1}$ is non-empty. There exist non-empty subsets $I_{1}, \ldots, I_{l}$ of $\llbracket 1, n \rrbracket$ such that $S_{1}=A_{I_{1}} \cap \cdots \cap A_{I_{l}}$.

As $B_{I}^{0} \bigcap S_{1}$ is non-empty, we have

$$
I \bigcap\left(I_{1} \bigcup \cdots \cup I_{l}\right)=\emptyset \text {. }
$$


Then, in $\mathbb{A}^{n}$, we have a neighbourhood $V_{0}$ of $B_{I}^{0} \bigcap S_{1}$ isomorphic to a product $\mathbb{A}^{d} \times \mathbb{A}^{|I|}$ with $d=n-|I|$ :

$$
\begin{array}{ccc}
\mathbb{A}^{d} & \times & \mathbb{A}^{|I|} \\
\cup & \cup \\
\tilde{D}_{d}^{1} & & \bigcup_{i \in I} \tilde{B}_{i}^{0},
\end{array}
$$

where $\tilde{B}_{i}^{0}$ is the hyperplane corresponding to $\left\{x_{i}=0\right\}$ inside $\mathbb{A}^{|I|}$.

Lifting this neighbourhood to $\widehat{V_{0}}$ in $X_{n}$, it becomes isomorphic to $X_{d} \times \mathbb{A}^{|I|}$ with $\widehat{D}_{d}^{1} \subset X_{d}$. Then, for any $\widehat{S}_{i}$ there is a stratum $\widehat{S_{i}^{d}}$ of $\widehat{D}_{d}^{1}$ such that $\widehat{V_{0}} \cap \widehat{S_{i}} \simeq \widehat{S_{i}^{d}} \times \mathbb{A}^{|I|}$. As the $\widehat{S_{i}^{d}}$ 's give a normal crossing divisor in $X_{d}$ by Theorem $4.2, \widehat{V_{0}}$ gives the neighbourhood of $\widehat{B_{I}^{0}} \cap \widehat{S_{1}} \cap \cdots \cap \widehat{S_{k}}$ such that $V \cap \widehat{D}_{n}$ is a normal crossing divisor in $X_{n}$.

Definition 4.11. Let $\widehat{B}_{n}$ denote the preimage of $B_{n}$ and $\widehat{A}_{n}$ the divisor $\widehat{D}_{n} \backslash \widehat{B}_{n}$.

Remark 4.12. The divisors $\widehat{A}_{n}$ and $\widehat{B}_{n}$ do not share any irreducible components and are both normal crossing divisors.

Let $\widehat{C}_{n}$ be the preimage of $C_{n}=[0,1]^{n}$ in $X_{n}$ and $\overline{\widehat{C}_{n}}$ its closure. Then $\widehat{B}_{n}$ is the Zariski closure of the boundary of $\widehat{C}_{n}$, and there is a non-zero class

$$
\left[\widehat{C}_{n}\right] \in \mathrm{Gr}_{0}^{W} \mathrm{H}^{n}\left(X_{n}, \widehat{B}_{n}\right) .
$$

If $I$ is a subset of $\llbracket 1, n \rrbracket$, we define $F_{I}$ and $G_{I}$ to be the functions

$$
\begin{aligned}
& G_{I}:\left(x_{1}, \ldots, x_{n}\right) \longmapsto \prod_{i \in I} x_{i} \\
& F_{I}:\left(x_{1}, \ldots, x_{n}\right) \longmapsto 1-\prod_{i \in I} x_{i} .
\end{aligned}
$$

Definition 4.13. A flag $\mathcal{F}$ of $\llbracket 1, n \rrbracket$ is a collection of non-empty distinct subsets $I_{j}$ of $\llbracket 1, n \rrbracket$ such that $I_{1} \subsetneq \ldots \subsetneq I_{r}$. The length of the flag $\mathcal{F}$ is the integer $r$ and we may say that $\mathcal{F}$ is an $r$-flag of $\llbracket 1, n \rrbracket$. A flag of length $n$ will be a maximal flag. A distinguished $r$-flag $\left(\mathcal{F}, i_{1}, \ldots i_{p}\right)$ will be a flag $\mathcal{F}$ of length $r$ together with elements $i_{1}<\ldots<i_{p}$ of $\llbracket 1, r \rrbracket$.

Definition 4.14. Let $\left(\mathcal{F}, i_{1}, \ldots i_{p}\right)$ be a distinguished $r$-flag of $\llbracket 1, n \rrbracket$. Let $\Omega_{i_{1}, \ldots, i_{p}}^{\mathcal{F}}$ denote the differential form of $\Omega_{l o g}^{\bullet}\left(\mathbb{A}^{n} \backslash D_{n}\right)$ defined by

$$
\Omega_{i_{1}, \ldots i_{p}}^{\mathcal{F}}=\bigwedge_{j=1}^{r} \mathrm{~d} \log \left(g_{j}\right)
$$

where

$$
g_{j}=\left\{\begin{array}{l}
F_{I_{j}} \text { if } j \in\left\{i_{1}, \ldots, i_{p}\right\} \\
G_{I_{j}} \text { otherwise. }
\end{array}\right.
$$

Let $\mathbf{k}=\left(k_{1}, \ldots, k_{p}\right)$ be a tuple of positive integers with $k_{1} \geqslant 2$ such that $k_{1}+$ $\cdots+k_{p}=n$, and let $s$ be a permutation of $\llbracket 1, n \rrbracket$. We define a differential form $\Omega_{\mathbf{k}, s} \in \Omega_{l o g}^{n}\left(\mathbb{A}^{n} \backslash D_{n}\right)$ by

$$
\Omega_{\mathbf{k}, s}=f_{k_{1}, \ldots, k_{n}}\left(x_{s(1)}, \ldots, x_{s(n)}\right) \mathrm{d} x_{1} \wedge \cdots \wedge \mathrm{d} x_{n} .
$$

Remark 4.15. Let $\mathbf{k}$ and $s$ be as in the previous definition. We associate to the pair $(\mathbf{k}, s)$ the maximal distinguished flag $\left(F_{k}, i_{1}, \ldots, i_{p}\right)$ defined by $I_{i}=\{s(1), \ldots, s(i)\}$ and $i_{j}=k_{1}+\cdots+k_{j}$ where $j$ varies from 1 to $p$. Then we can see that there exists an integer $r_{s}$ such that

$$
\Omega_{\mathbf{k}, s}=(-1)^{r_{s}} \Omega_{i_{1}, \ldots i_{p}}^{\mathcal{F}_{k}} .
$$


Definition 4.16. We shall write $\omega_{i_{1}, \ldots, i_{p}}^{\mathcal{F}}$ and $\omega_{\mathbf{k}, s}$ for the pull back on $X_{n} \backslash \widehat{D}_{n}$ of the forms $\Omega_{I_{1}, \ldots, I_{p}}$ and $\Omega_{\mathbf{k}, s}$, respectively.

Proposition 4.17. If $\left(\mathcal{F}, i_{1}, \ldots, i_{p}\right)$ is a maximal flag of $\llbracket 1, n \rrbracket$ such that $i_{1} \geqslant 2$ and $i_{p}=n$ then:

- The divisor of singularities $A_{i_{1}, \ldots, i_{p}}^{\mathcal{F}}$ of $\Omega_{i_{1}, \ldots, i_{p}}^{\mathcal{F}}$ is $A_{I_{i_{1}}} \cup \cdots \cup A_{I_{i_{p}}}$.

- The divisor of singularities $\widehat{A}_{i_{1}, \ldots, i_{p}}^{\mathcal{F}}$ of $\omega_{i_{1}, \ldots, i_{p}}^{\mathcal{F}}$ lies in $\widehat{A}_{n}$. Thus, the divisor of singularities of $\omega_{\mathbf{k}, s}$ lies in $\widehat{A}_{n}$.

Moreover, let $\left(\mathcal{F}, i_{1}, \ldots, i_{p}\right)$ and $\left(\mathcal{F}^{\prime}, i_{1}^{\prime}, \ldots, i_{q}^{\prime}\right)$ be two distinguished flags such that the length of $\mathcal{F}$ (resp. $\left.\mathcal{F}^{\prime}\right)$ is $i_{p}$ (resp $i_{q}^{\prime}$ ) with $\left|I_{i_{1}}\right| \geqslant 2$ (resp. $\left|I_{i_{1}^{\prime}}^{\prime}\right| \geqslant 2$ ) and suppose that the sets $I_{i_{p}}$ and $I_{i_{q}^{\prime}}^{\prime}$ form a partition of $\llbracket 1, n \rrbracket$. Then the divisor of singularities of $\omega_{i_{1}, \ldots, i_{p}}^{\mathcal{F}} \wedge \omega_{i_{1}^{\prime}, \ldots, i_{q}^{\prime}}^{\mathcal{F}^{\prime}}$ lies in $\widehat{A}_{n}$.

Let $\left(\mathcal{F}, i_{1} \subsetneq \ldots \subsetneq i_{p}\right)$ be a flag as in the previous proposition.

It is straightforward to see that $A_{I_{i_{1}}, \ldots I_{i_{p}}}^{\mathcal{F}}$ is $A_{I_{i_{1}}} \cup \cdots \cup A_{I_{i_{p}}}$. The following lemma due to Goncharov can easily be modified to fit into our situation.

Lemma 4.18 ([Gon02][lemma 3.8]). Let $Y$ be a normal crossing divisor in a smooth variety $X$ and $\omega \in \Omega_{\text {log }}^{n}(X \backslash Y)$. Let $p: \widehat{X} \longrightarrow X$ be the blow-up of an irreducible variety $Z$. Suppose that the generic point of $Z$ is different from the generic points of strata of $Y$. Then $p^{*} \omega$ does not have a singularity at the special divisor of $\widehat{X}$.

The modified version we use here is given in the following statement.

Lemma 4.19. Let $Y$ be a normal crossing divisor in $\mathbb{A}^{n}$ and $\omega \in \Omega_{\text {log }}^{n}\left(\mathbb{A}^{n} \backslash Y\right)$. Let $p_{n}: X_{n} \rightarrow \mathbb{A}^{n}$ be the map of our previous construction. Suppose that the generic points of the strata of $B_{n}$ that are blown up in the construction of $X_{n}$ are different from the generic points of strata of $Y$. Then $p_{n}^{*} \omega$ does not have singularities at the corresponding exceptional divisors in $\widehat{B}_{n}$.

It is enough to check that the divisor of singularities of $\Omega_{i_{1}, \ldots, i_{P}}^{\mathcal{F}}$ is a a normal crossing divisor, and that none of its strata is a blown up strata of $B_{n}$.

The divisor of singularities of $\Omega_{i_{1}, \ldots, i_{p}}^{\mathcal{F}}$ is $A_{I_{i_{1}}} \cup \cdots \cup A_{I_{i_{p}}}$, and to show it is a normal crossing divisor, it is enough to show that the normal vectors of the $A_{I_{i_{j}}}$ at any intersection of some of them are linearly independent. The normal vector of $A_{I_{i_{j}}}$ is $\sum_{i \in I_{i_{j}}} 1 / x_{i} \mathrm{~d} x_{i}$, and as we have $I_{1} \subsetneq I_{2} \subsetneq \ldots \subsetneq I_{p}$, they are linearly independent.

We now have to show that none of the strata of $B_{n}$ that are blown up in the construction of $X_{n}$ are exactly some strata of $A_{I_{1}} \cup \cdots A_{I_{p}}$. Let $S$ be such a stratum of $B_{n}$ of codimension $k$. The stratum $S$ is defined by the equations $x_{r_{1}}=$ $1, \ldots, x_{r_{k}}=1$. If $I_{S}$ denotes the set $\left\{r_{1}, \ldots, r_{k}\right\}$, then for any subset $I$ of $\llbracket 1, n \rrbracket$, $S$ is included in $A_{I}$ if and only $I$ is included in $I_{S}$. As $I_{i} \subset I_{i^{\prime}}$ for $i<i^{\prime}$, if $S$ is included in a stratum $S_{A}$ of $A_{I_{1}, \ldots, I_{p}}$, that stratum is of the form $A_{I_{i_{1}}} \cap \cdots \cap A_{A_{i_{j}}}$ with $j<k$ because $\left|I_{1}\right| \leqslant 2$. As a consequence, $S_{A}$ is of codimension at most $k-1$, and $S$ cannot be a stratum of $A_{i_{1}, \ldots, i_{p}}^{\mathcal{F}}$.

For the case of two distinguished flags, we use the same argument as in the lemma, so the proposition 4.17 is proved.

Proposition 4.20. The divisor $\widehat{A}_{n}$ does not intersect the boundary of $\widehat{C}_{n}$ in $X_{n}(R)$.

Proof. Let $S$ be an irreducible codimension 1 stratum of $\widehat{B}_{n}$ containing an intersection of some strata of $\widehat{A}_{n}$ with the boundary of $\bar{C}_{n}$. As the divisor $A_{n}$ intersects the boundary of the real cube $C_{n}$ only on strata of $B_{n}$ that are of codimension at least $2, S$ has to be such that $p_{n}(S)$ is a stratum of $B_{n}$ of codimension at least 2 . 
Using the symmetry with respect to the standard coordinates on $\mathbb{A}^{n}$, we can suppose that $p_{n}(S)$ is defined in those coordinates by $x_{k}=x_{k+1}=\ldots=x_{n}$.

Starting from $\mathbb{A}^{n}$ and blowing up first the point $x_{1}=x_{2}=\ldots=x_{n}=1$, then the edge $x_{2}=x_{3}=\ldots=x_{n}=1$ and after that the plane $x_{3}=x_{4}=\ldots=x_{n}=1$ and so on, we obtain a variety $\widetilde{p}_{n}: \widetilde{X}_{n} \rightarrow \mathbb{A}^{n}$. There are natural local coordinates $\left.\left(s_{1}, \ldots, s_{n}\right)\right)$ on $\widetilde{X}_{n}$ such that the coordinates on $\mathbb{A}^{n}$ defined by $y_{i}=1-x_{i}$ satisfy:

$$
y_{1}=s_{1}, \quad y_{2}=s_{1} s_{2}, \quad \ldots, \quad y_{i}=s_{1} s_{2} \cdots s_{i}, \quad \ldots, \quad y_{n}=s_{1} s_{2} \cdots s_{n} .
$$

In the $y_{i}$-coordinates, the stratum $x_{j}=x_{j+1}=\ldots=x_{n}=1$ is given by

$$
y_{j}=y_{j+1}=\ldots=y_{n}=0
$$

and its preimage in $\widetilde{X}_{n}$ is given by $s_{j}=0$.

For any permutation $s$ of $\llbracket 1, n \rrbracket$, we could apply the same construction, that is blowing up the point $x_{s(1)}=x_{s(2)}=\ldots=x_{s(n)}=1$ then the edge $x_{s(2)}=x_{s(3)}=$ $\ldots=x_{s(n)}=1$ and so on, and obtain a variety $\widetilde{p}_{n}^{s}: \widetilde{X}_{n}^{s} \rightarrow \mathbb{A}^{n}$. The preimage of $D_{n}$ in $\widetilde{X}_{n}^{s}$ will be denoted by $\widetilde{D}_{n}^{s}, \widetilde{B}_{n}^{s}$ will denote the preimage of $B_{n}$ and $\widetilde{A}_{n}^{s}$ is $\widetilde{D}_{n}^{s} \backslash \widetilde{B}_{n}^{s}$. To prove that $\widehat{A}_{n}$ does not intersect the boundary of $\widehat{C}_{n}$ in $X_{n}(\mathbb{R})$, it is enough to show that for any permutation $s, \widetilde{A}_{n}^{s}$ does not intersect, in $\widetilde{X}_{n}^{s}(\mathbb{R})$, the boundary of the preimage of $C_{n}$. It is then enough to show that the proper transforms of the divisors $A_{I}$ do not intersect the boundary of $\tilde{X}_{n}^{s}(\mathbb{R})$, because it will then be the same for the irreducible components of their intersections as for the proper transforms of those components by the remaining blow-up used to reach $X_{n}$. By symmetry, it is enough to show it when $s$ is the identity map and then in the case of $\widetilde{X}_{n}$. Let $\widetilde{C}_{n}$ be the preimage of $C_{n}$ in $\widetilde{X}_{n}$.

Let $A_{I}$ be a codimension 1 stratum of $A_{n}$, where $I$ is the set $\left\{i_{0}, \ldots, i_{p}\right\}$ and suppose that $i_{0}<\ldots<i_{p}$. We want to show that the closure $\widetilde{A}_{I}$ of the preimage of $A_{I} \backslash B_{n}$ in $\widetilde{X}_{n}$ does not intersect the boundary of $\widetilde{C}_{n}$. The $k$-th symmetric function will be denoted by $\sigma_{k}$ with the following convention

$$
\sigma_{0}=1, \quad \sigma_{k}\left(X_{1}, \ldots, X_{l}\right)=0 \text { if } l>k .
$$

The stratum $A_{I}$ is defined in the $x_{i}$-coordinates by $1-x_{i_{0}} \cdots x_{i_{p}}=0$ and in the $y_{i}$ coordinates by

$$
0=\sum_{k=1}^{p+1}(-1)^{k-1} \sigma_{k}\left(y_{i_{0}}, y_{i_{1}} \ldots, y_{i_{p}}\right) .
$$

Before giving an explicit expression of $\widetilde{A}_{I}$ with the $s_{i}$ coordinates, we define the set $J_{0}$ as $\left\{1, \ldots, i_{0}\right\}$ and the sets $J_{1}, \ldots, J_{p}$ by

$$
J_{k}=\left\{i_{0}+1, i_{0}+2, \ldots, i_{k}\right\}
$$

for all $k$ in $\llbracket 1, p \rrbracket$.

For any subset $J$ of $\llbracket 1, n \rrbracket, \Pi^{J} \mathbf{s}$ will denote the product $\prod_{j \in J} s_{j}$. We have the following relations

$$
y_{i_{0}}=\Pi^{J_{0}} \mathbf{s} \quad \text { and } \quad \forall k \in \llbracket 1, p \rrbracket, \quad y_{i_{k}}=\Pi^{J_{0}} \mathbf{s} \Pi^{J_{k}} \mathbf{s} .
$$

Using the variable change $y_{i}=s_{1} \cdots s_{i}$, the RHS of the equation (20) can be written

$$
\sum_{k=1}^{p+1}(-1)^{k-1} \sigma_{k}\left(\Pi^{J_{0}} \mathbf{s}, \Pi^{J_{0}} \mathbf{s} \Pi^{J_{1}} \mathbf{s}, \ldots, \Pi^{J_{0}} \mathbf{s} \Pi^{J_{p}} \mathbf{s}\right) .
$$

For any indeterminate $\lambda$ and any $k$, one has

$$
\sigma_{k}\left(\lambda, \lambda X_{1}, \lambda X_{2}, \ldots, \lambda X_{p}\right)=\lambda^{k}\left(\sigma_{k-1}\left(X_{1} \ldots, X_{p}\right)+\sigma_{k}\left(X_{1}, \ldots, X_{p}\right)\right) .
$$


Then the expression (21) is equal to

$$
\begin{aligned}
& \Pi^{J_{0}} \mathbf{s}\left[1+\sigma_{1}\left(\Pi^{J_{1}} \mathbf{s}, \ldots, \Pi^{J_{p}} \mathbf{s}\right)\right. \\
&+\sum_{k=1}^{p-1}\left((-1)^{k}\left(\Pi^{J_{0}} \mathbf{s}\right)^{k}\left(\sigma_{k}\left(\Pi^{J_{1}} \mathbf{s}, \ldots, \Pi^{J_{p}} \mathbf{s}\right)+\sigma_{k+1}\left(\Pi^{J_{1}} \mathbf{s}, \ldots, \Pi^{J_{p}} \mathbf{s}\right)\right)\right) \\
&\left.+(-1)^{p} \sigma_{p}\left(\Pi^{J_{1}} \mathbf{s}, \ldots, \Pi^{J_{p}} \mathbf{s}\right)\right] .
\end{aligned}
$$

The expression of $\widetilde{A}_{I}$ in the $s_{i}$-coordinates is then

$$
\begin{aligned}
& 0=1+\sigma_{1}\left(\Pi^{J_{1}} \mathbf{s}, \ldots, \Pi^{J_{p}} \mathbf{s}\right) \\
&+\sum_{k=1}^{p-1}\left((-1)^{k}\left(\Pi^{J_{0}} \mathbf{s}\right)^{k}\left(\sigma_{k}\left(\Pi^{J_{1}} \mathbf{s}, \ldots, \Pi^{J_{p}} \mathbf{s}\right)+\sigma_{k+1}\left(\Pi^{J_{1}} \mathbf{s}, \ldots, \Pi^{J_{p}} \mathbf{s}\right)\right)\right) \\
&+(-1)^{p} \sigma_{p}\left(\Pi^{J_{1}} \mathbf{s}, \ldots, \Pi^{J_{p}} \mathbf{s}\right) .
\end{aligned}
$$

The closure of $\widetilde{C}_{n}$ is given, in the $s_{i}$ coordinates, by $s_{1} \in[0,1]$, and for any $i \in \llbracket 1, n \rrbracket$ by $s_{1} \cdots s_{i} \in[0,1]$. It is enough to look the intersection of $\widetilde{A}_{I}$ with codimension 1 strata of the boundary of $\widetilde{C}_{n}$.

Suppose that $s_{i_{0}}=0$ for some $i_{0} \in J_{0}$. Then the RHS of (22) becomes

$$
1+\sigma_{1}\left(\Pi^{J_{1}} \mathbf{s}, \ldots, \Pi^{J_{p}} \mathbf{s}\right)
$$

which is strictly positive if $s_{i} \geqslant 0$ for any $i$. So the divisor $\widetilde{A}_{I}$ does not intersect any component of the form $s_{i_{0}}=0$ for $i_{0}$ in $J_{0}$.

Then, we can suppose that $s_{i} \neq 0$ for all $i \in J_{0}$ in order to study the intersection of $\widetilde{A}_{I}$ with the boundary of $\widetilde{C}_{n}$, and the RHS of (22) can be written

$$
\frac{1}{\Pi^{J_{0}} \mathbf{s}}\left(1-\prod_{j=1}^{p}\left(1-\Pi^{J_{0}} \mathbf{s} \Pi^{J_{j}} \mathbf{s}\right)\right)+\prod_{j=1}^{p}\left(1-\Pi^{J_{0}} \mathbf{s} \Pi^{J_{j}} \mathbf{s}\right) .
$$

Suppose that a point $x=\left(s_{1}, \ldots, s_{n}\right)$ with $s_{i}>0$ for all $i$ in $J_{0}$, lies in the closure of $\widetilde{C}$. That is, for all $i$ in $\llbracket 1, n \rrbracket$, the product $s_{1} s_{2} \cdots s_{i}$ is between 0 and 1 which means all the products $\Pi^{J_{0}} \mathbf{s} \Pi^{J_{j}} \mathbf{s}$ lie between 0 and 1 for $j$ in $\llbracket 1, p \rrbracket$. Then one find the following inequalities

$$
\begin{aligned}
0 \leqslant \frac{1}{\Pi^{J_{0}} \mathbf{s}} & \left(1-\prod_{j=1}^{p}\left(1-\Pi^{J_{0}} \mathbf{s} \Pi^{J_{j}} \mathbf{s}\right)\right) \leqslant \frac{1}{\Pi^{J_{0}} \mathbf{s}} \\
0 & \leqslant \prod_{j=1}^{p}\left(1-\Pi^{J_{0}} \mathbf{s} \Pi^{J_{j}} \mathbf{s}\right) \leqslant 1 .
\end{aligned}
$$

Both terms cannot simultaneously be equal to 0 , thus $\widetilde{A}_{I}$ does not intersect the boundary of $\widetilde{C}_{n}$ since the $s_{i}$ are strictly positive for $i$ in $J_{0}$ and the proposition is proved.

4.3. An alternative definition for motivic MZV. Both propositions 4.17 and 4.20 lead to the following theorem and to an alternative definition for motivic multiple zeta values. 
Theorem 4.21. Let $\mathbf{k}=\left(k_{1}, \ldots, k_{p}\right)$ be a tuple of integers with $k_{1} \geqslant 2$ and $k_{1}+\ldots+$ $k_{p}=n$, and let $s$ be a permutation of $\llbracket 1, n \rrbracket$. Let $\widehat{A}_{\mathbf{k}}^{s}$ be the divisor of singularities of the differential form $\omega_{\mathbf{k}}^{s}$. Then there exists a mixed Tate motive

$$
\mathrm{H}^{n}\left(X_{n} \backslash \widehat{A}_{\mathbf{k}}^{s} ; \widehat{B}_{n}^{\widehat{A}_{\mathbf{k}}^{s}}\right) .
$$

The differential form $\omega_{\mathbf{k}}^{s}$ and the preimage $\widehat{C}_{n}$ of the real $n$-dimensional cube in $X_{n}$ give two non zero elements

$$
\left[\omega_{\mathbf{k}}^{s}\right] \in \operatorname{Gr}_{2 n}^{W} \mathrm{H}^{n}\left(X_{n} \backslash \widehat{A}_{\mathbf{k}}^{s} ; \widehat{B}_{n}^{\widehat{A}_{\mathbf{k}}^{s}}\right) \quad \text { and } \quad\left[\widehat{C}_{n}\right] \in\left(\operatorname{Gr}_{0}^{W} \mathrm{H}^{n}\left(X_{n} \backslash \widehat{A}_{\mathbf{k}}^{s} ; \widehat{B}_{n}^{\widehat{A}_{\mathbf{k}}^{s}}\right)\right)^{\vee}
$$

The period of the $n$-framed mixed Tate motive

is equal to $\zeta\left(k_{1}, \ldots, k_{n}\right)$.

$$
\zeta^{f r ., \mathcal{M}}(\mathbf{k}, s)=\left[\mathrm{H}^{n}\left(X_{n} \backslash \widehat{A}_{\mathbf{k}}^{s} ; \widehat{B}_{n}^{\widehat{A}_{\mathbf{k}}^{s}}\right) ;\left[\omega_{\mathbf{k}}^{s}\right],\left[\widehat{C}_{n}\right]\right]
$$

Moreover, let $\left(\mathcal{F}, i_{1}, \ldots, i_{p}\right)$ and $\left(\mathcal{F}^{\prime}, i_{1}^{\prime}, \ldots, i_{q}^{\prime}\right)$ be two distinguished flags such that the length of $\mathcal{F}$ (resp. $\left.\mathcal{F}^{\prime}\right)$ is $i_{p}$ (resp. $i_{q}^{\prime}$ ) with $\left|I_{i_{1}}\right| \geqslant 2$ (resp. $\left|I_{i_{1}^{\prime}}^{\prime}\right| \geqslant 2$ ) and the sets $I_{i_{p}}, I_{i_{q}}^{\prime}$ form a partition of $\llbracket 1, n \rrbracket$ and let $\widehat{A}_{i_{1}, \ldots, l_{p} \mid i_{1}^{\prime}, \ldots, i_{q}^{\prime}}^{\mathcal{F}}$ be the divisor of singularities of $\omega_{i_{1}, \ldots, i_{p}}^{\mathcal{F}} \wedge \omega_{i_{1}^{\prime}, \ldots, i_{q}^{\prime}}^{\mathcal{F}^{\prime}}$. There exists an $n$-framed mixed Tate motive

$$
\zeta^{f r ., \mathcal{M}}\left(\mathcal{F}, i_{1}, \ldots, i_{p} \mid \mathcal{F}^{\prime}, i_{1}^{\prime}, \ldots, i_{q}^{\prime}\right)=\mathrm{H}^{n}\left(X_{n} \backslash \widehat{A}_{i_{1}, \ldots, l_{p} \mid i_{1}^{\prime}, \ldots, i_{q}^{\prime}}^{\mathcal{F} \mid \mathcal{F}^{\prime}} ; \widehat{B}_{n}^{\widehat{A}_{i_{1}, \ldots, l_{p} \mid i_{1}^{\prime}, \ldots, i_{q}^{\prime}}^{\mathcal{F}}}\right),
$$

the frames being given by $\left[\omega_{i_{1}, \ldots, i_{p}}^{\mathcal{F}} \wedge \omega_{i_{1}^{\prime}, \ldots, i_{q}^{\prime}}^{\mathcal{F}^{\prime}}\right]$ and $\left[\widehat{C}_{n}\right]$.

Proof. We want to apply theorem 3.6 in [Gon02] to our particular case. As $\widehat{D}_{n}$ is a normal crossing divisor and as proposition 4.20 ensures that $\widehat{A}_{n}$ does not intersect $\left[\widehat{C}_{n}\right]$, using Proposition 4.17 , the only thing that remains to show is that we have a Tate stratification of $X_{n}$, which is ensured by Lemma 4.23.

The computation of the period follows from the fact that integrating over $\widehat{C}_{n}$ is the same as integrating over the real cube.

The key to prove Lemma 4.23 is Lemma 4.7 from which we deduce the following lemma.

Lemma 4.22. Let $I_{1}, \ldots, I_{k}$ be $k$ subsets of $\llbracket 1, n \rrbracket$ and let $X$ be the intersection $A_{I_{1}} \cap \cdots \cap A_{I_{k}} \subset \mathbb{A}^{n}$. Then $X$ and its irreducible components are Tate varieties.

Proof. Using Lemma 4.7, we have non negative integers $r$ and $s$ and integers $c_{1}, \ldots, c_{r}$ such that there exists an isomorphism $f$

$$
X \longrightarrow \mathbb{A}^{s} \times \mathbb{G}_{m}^{n-s-r} \times \prod_{i=1}^{r}\left\{x^{c_{i}}=1\right\} .
$$

Moreover, there is a one to one map between the set of the irreducible components of $X$ and the set of those of $\prod_{i=1}^{r}\left\{x^{c_{i}}=1\right\}$; the irreducible components of $X$ are thus disjoint.

We conclude that the motive of $X$ is a direct sum of Tate motives, in other words $X$ is a Tate variety. The irreducible components of $X$ being disjoint, each is a Tate variety.

Lemma 4.23. The divisor $\widehat{D}_{n}=\widehat{B}_{n}^{0} \cup \widehat{D}_{n}^{1}$ provides $X_{n}$ with a Tate stratification.

Proof. We first need to show that all the strata of $\widehat{D}_{n}^{1}$ and $X_{n}$ are Tate, but using Proposition 4.4 , it is enough to show that all the strata of $D_{n}^{1}$ are Tate $\left(\mathbb{A}^{n}\right.$ being 
Tate). A stratum $A_{I_{1}} \cap \cdots \cap A_{I_{k}}$ of $D_{n}^{1}$ is a Tate variety by Lemma 4.22 . So $X_{n}$ and all the strata of $\widehat{D}_{n}^{1}$ are Tate.

Note that the previous discussion tells us that for any $k \geqslant 2, X_{k}$ and all the strata of $\widehat{D}_{k}^{1}$ are Tate varieties.

Let $\widehat{S}$ be the intersection of certain codimension 1 strata of $\widehat{B}_{n}^{0}$; it is the proper transform of the corresponding intersection, say $S=\cap_{j \in J}\left\{x_{j}=0\right\}$ for some $J \subset$ $\llbracket 1, n \rrbracket$, in $B_{n}^{0}$. That is, $\widehat{S}$ is isomorphic to

$$
\mathrm{Bl}_{S \cap D: D \in \mathcal{D}_{n}^{1}} S \text {. }
$$

The intersection $S$ is isomorphic to $\mathbb{A}^{d}$ for $d=n-|J|$ and hence is Tate, and if $I$ is a subset of $\llbracket 1, n \rrbracket$, then $S \cap A_{I}$ is either empty $(I \cap J \neq \emptyset)$ or, if $I \cap J=\emptyset$, isomorphic to the subvariety of $\mathbb{A}^{d}$ given by $\left\{1-\prod_{i \in I} x_{i}=0\right\}$ (up to renumbering). Thus, the proper transform $\widehat{S}$ is isomorphic to $X_{d}$, which is Tate by the discussion above.

Now, if $\widehat{S}_{i}$ is some irreducible codimension 1 stratum of $\widehat{D}_{n}^{1}$ that has a non-empty intersection with $\widehat{S}$, then, as $\widehat{S}_{i}$ is the exceptional divisor of some of the blow-ups in the construction of $X_{n}$, this intersection $\widehat{S} \cap \widehat{S}_{i}$ is the exceptional divisor in the blow-up sequence (23) that leads to $\widehat{S}$. As a consequence, the intersection $\widehat{S} \cap \widehat{S}_{i}$ is isomorphic to some irreducible stratum of $\widehat{D}_{d}^{1}$ in $X_{d}$ and we can conclude that any possible intersection of strata in $\widehat{D}_{n}^{1}$ with $\widehat{S}$ is isomorphic to an intersection of strata in $\widehat{D}_{d}^{1}$ inside $X_{d} \simeq \widehat{S}$, and so is Tate by the above discussion.

4.4. Motivic Stuffle. Let $\mathbf{k}=\left(k_{1}, \ldots, k_{p}\right)$ and $\mathbf{l}=\left(l_{1}, \ldots, l_{q}\right)$ be respectively a $p$-tuple and a $q$-tuple of integers with $k_{1}, l_{1} \geqslant 2, \sum k_{i}=n$ and $\sum l_{j}=m$. In this section, as in section 1.1 and 1.3, if $\sigma$ is a term of the formal $\operatorname{sum~} \mathbf{k} * \mathbf{l}$ with all coefficients being equal to 1 , we will write $\sigma \in \operatorname{st}(\mathbf{k}, \mathbf{l})$. The map $\delta$ defined in Proposition 2.3 extends to:

$$
\overline{\mathcal{M}_{0, n+m+3}} \stackrel{\delta}{\longrightarrow} \overline{\mathcal{M}_{0, n+3}} \times \overline{\mathcal{M}_{0, m+3}} .
$$

Let $A_{\mathbf{k}}$ (resp. $A_{\mathbf{l}}$ ) be the divisor of singularities of the meromorphic differential form $\omega_{\mathbf{k}}$ on $\overline{\mathcal{M}_{0, n+3}}$ (resp. $\omega_{\mathbf{l}}$ on $\overline{\mathcal{M}_{0, m+3}}$ ) given in simplicial coordinates by $\omega_{\bar{k}}$ (resp. $\omega_{\bar{l}}$ ) (cf. 3) and given in the cubical coordinates by $f_{k_{1}, \ldots, k_{p}}\left(\right.$ resp. $\left.f_{l_{1}, \ldots, l_{q}}\right)$. For all $\sigma$ in $\operatorname{st}(\mathbf{k}, \mathbf{l})$, let $A_{\sigma}$ be the divisor of singularities of the form $\omega_{\sigma}$. As in section 3.2 , let $\Phi_{n}, \Phi_{m}$ and $\Phi_{n+m}$ denote respectively the standard cells in $\overline{\mathcal{M}_{0, n+3}}(\mathbb{R})$, $\overline{\mathcal{M}_{0, m+3}}(\mathbb{R})$ and $\overline{\mathcal{M}_{0, n+m+3}}(\mathbb{R})$ and $B_{n}, B_{m}$ and $B_{n+m}$ be the Zariski closure of the boundary of $\Phi_{n}, \Phi_{m}$ and $\Phi_{n+m}$ respectively.

Proposition 4.24. We have an equality of framed motives:

$$
\begin{array}{r}
{\left[\mathrm{H}^{n}\left(\overline{\mathcal{M}_{0, n+3}} \backslash A_{\mathbf{k}} ; B_{n}^{A_{\mathbf{k}}}\right) ;\left[\omega_{\mathbf{k}}\right] ;\left[\Phi_{n}\right]\right] \cdot\left[\mathrm{H}^{m}\left(\overline{\mathcal{M}_{0, m+3}} \backslash A_{\mathbf{l}} ; B_{m}^{A_{1}}\right) ;\left[\omega_{\mathbf{l}}\right] ;\left[\Phi_{m}\right]\right]=} \\
\sum_{\sigma \in \operatorname{st}(\mathbf{k}, \mathbf{l})}\left[\mathrm{H}^{n+m}\left(\overline{\mathcal{M}_{0, n+m+3}} \backslash A_{\sigma} ; B_{n+m}^{A_{\sigma}}\right) ;\left[\omega_{\sigma}\right] ;\left[\Phi_{n+m}\right]\right] .
\end{array}
$$

Proof. Let $A_{0}$ be the Zariski closure of $\partial \overline{\mathcal{M}_{0, n+m+3}} \backslash B_{n+m}, B_{n, m}$ the Zariski closure of the boundary of $\Phi_{n} \times \Phi_{m}$ and $A^{\prime}$ the boundary of $\left(\overline{\mathcal{M}_{0, n+3}} \backslash A_{\mathbf{k}}\right) \times$ $\left(\overline{\mathcal{M}_{0, m+3}} \backslash A_{\mathbf{l}}\right)$. As the map $\delta$ maps $B_{n+m}$ onto $B_{n, m}$, we have an induced map

$$
\left.\delta:\left(\overline{\mathcal{M}_{0, n+m+3}} \backslash A_{0} ; B_{n+m}^{A_{0}}\right) \longrightarrow\left(\overline{\mathcal{M}_{0, n+3}} \backslash A_{\mathbf{k}}\right) \times\left(\overline{\mathcal{M}_{0, m+3}} \backslash A_{\mathbf{l}}\right) ; B_{n, m}^{A^{\prime}}\right) .
$$


Using the Künneth formula, we have maps of mixed Tate motives

$$
\begin{gathered}
\mathrm{H}^{n}\left(\overline{\mathcal{M}_{0, n+3}} \backslash A_{\mathbf{k}} ; B_{n}^{A_{\mathbf{k}}}\right) \otimes \mathrm{H}^{m}\left(\overline{\mathcal{M}_{0, m+3}} \backslash A_{\mathbf{l}} ; B_{m}^{A_{\mathbf{l}}}\right) \longrightarrow \\
\mathrm{H}^{n+m}\left(\left(\overline{\mathcal{M}_{0, n+3}} \backslash A_{\mathbf{k}}\right) \times\left(\overline{\mathcal{M}_{0, m+3}} \backslash A_{\mathbf{l}}\right) ; B_{n, m}^{A^{\prime}}\right) \longrightarrow \\
\mathrm{H}^{n+m}\left(\overline{\mathcal{M}_{0, n+m+3}} \backslash A_{0} ; B_{n+m}^{A_{0}}\right)
\end{gathered}
$$

which are both compatible with the respective frames $\left[\omega_{\mathbf{k}}\right] \otimes\left[\omega_{\mathbf{l}}\right] ;\left[\Phi_{n}\right] \otimes\left[\Phi_{m}\right]$, $\left[\omega_{\mathbf{k}} \wedge \omega_{\mathbf{l}}\right] ;\left[\Phi_{n} \times \Phi_{m}\right]$ and $\left[\delta^{*}\left(\omega_{\mathbf{k}} \wedge \omega_{\mathbf{l}}\right)\right] ;\left[\Phi_{n+m}\right]$.

We now need to show that

$$
\begin{aligned}
{\left[\mathrm{H}^{n+m}\left(\overline{\mathcal{M}_{0, n+m+3}} \backslash A_{0} ; B_{n+m}^{A_{0}}\right) ;\left[\delta^{*}\left(\omega_{\mathbf{k}} \wedge \omega_{\mathbf{l}}\right)\right],\left[\Phi_{n+m}\right]\right]=} \\
\quad \sum_{\sigma \in \operatorname{st}(\mathbf{k}, \mathbf{l})}\left[\mathrm{H}^{n+m}\left(\overline{\mathcal{M}_{0, n+m+3}} \backslash A_{\sigma} ; B_{n+m}^{A_{\sigma}}\right) ;\left[\omega_{\sigma}\right] ;\left[\Phi_{n+m}\right]\right] .
\end{aligned}
$$

As $A_{\sigma}$ is included in $A_{0}$, using lemma 3.6 it is enough to prove the previous equality with $A_{0}$ instead of $A_{\sigma}$ in the RHS. The two following lemmas tell us that it is enough to work with $X_{n+m}$ (cf. section 4.2) instead of $\overline{\mathcal{M}_{0, n+m+3}} \backslash A_{0}$.

Lemma 4.25. Let $r \geqslant 2$ be an integer and let $\tilde{\delta_{r}}: \overline{\mathcal{M}_{0, r+3}} \rightarrow\left(\mathbb{P}^{1}\right)^{r}$ be the map given on the open set by

$$
\begin{aligned}
\left(0, z_{1}, \ldots, z_{r}, 1, \infty\right) \longmapsto\left(0, z_{1}, z_{2}, \infty\right) \times\left(0, z_{2},\right. & \left.z_{3}, \infty\right) \times \cdots \\
& \times\left(0, z_{r-1}, z_{r}, \infty\right) \times\left(0, z_{r}, 1, \infty\right) .
\end{aligned}
$$

Let $A_{r}$ be the union of the codimension 1 irreducible components of $\partial \overline{\mathcal{M}_{0, r+3}}$ that are mapped by $\tilde{\delta_{r}}$ into $\left(\mathbb{P}^{1}\right)^{r} \backslash \mathbb{A}^{r}$.

Then, $A_{r} \subset A_{0}$ and there exists a sequence of flags $\mathcal{F}_{1}, \ldots, \mathcal{F}_{N}$ of elements of $\mathcal{D}_{r}^{1}$ (Lemma 4.9) satisfying conditions of Corollary 4.3 such that

$$
X_{r}=\mathrm{Bl}_{\mathcal{F}_{N}, \ldots, \mathcal{F}_{1}} \mathbb{A}^{r} \stackrel{\alpha_{r}}{\longrightarrow} \overline{\mathcal{M}_{0, r+3}} \backslash A_{r}=\mathrm{Bl}_{\mathcal{F}_{r}, \ldots, \mathcal{F}_{1}} \mathbb{A}^{r} \stackrel{\tilde{\delta_{r}}}{\longrightarrow} \mathbb{A}^{r} .
$$

Proof. The map $\tilde{\delta_{r}}$ is given in cubical coordinates on $\overline{\mathcal{M}_{0, r+3}}$ by $x_{i}=u_{i}$, where the $x_{i}$ denote the standard affine coordinates on $\left(\mathbb{P}^{1}\right)^{r}$. It maps $B_{r}$ into hyperplanes $x_{i}=0$ or $x_{i}=1$.

The induced map $\overline{\mathcal{M}_{0, r+3}} \backslash A_{r} \rightarrow \mathbb{A}^{r}$ is the blow-up along the strata

$$
\left\{x_{i}=x_{i+1}=\ldots=x_{j}=1\right\}
$$

which are all elements of $\mathcal{D}_{r}^{1}$.

The beginning $\mathcal{F}_{1}, \ldots, \mathcal{F}_{r}$ of the sequence of flags is given by

$$
\begin{gathered}
\mathcal{F}_{1}=\left\{\left\{x_{1}=x_{2}=\cdots=x_{n}=1\right\},\left\{x_{1}=x_{2}=\cdots=x_{n-1}=1\right\}, \ldots,\left\{x_{1}=1\right\}\right\} \\
\mathcal{F}_{2}=\left\{\left\{x_{2}=x_{3}=\cdots=x_{n}=1\right\},\left\{x_{2}=x_{3}=\cdots=x_{n-1}=1\right\}, \ldots,\left\{x_{2}=1\right\}\right\} \\
\ldots \\
\mathcal{F}_{i}=\left\{\left\{x_{i}=x_{i+1}=\cdots=x_{n}=1\right\},\left\{x_{i}=x_{i+1}=\cdots=x_{n-1}=1\right\}, \ldots,\left\{x_{i}=1\right\}\right\} \\
\cdots \\
\mathcal{F}_{r}=\left\{\left\{x_{r}=1\right\}\right\} .
\end{gathered}
$$

This part of the sequence satisfies condition (2) of Corollary 4.3. Then the easiest way to complete the sequence is to take flags with just one element beginning with the rank 1 strata of $\mathcal{D}_{r}^{1}$ (the only stratum of rank 0 is $\left\{x_{1}=x_{2}=\ldots=x_{n}=1\right\}$ ), then the rank 2 strata and so on.

Now that the sequence of flags exists, Corollary 4.3 ensures that the morphisms in (25) are well-defined. 
Indeed, the usual map $\overline{\mathcal{M}_{0, r+3}} \rightarrow\left(\mathbb{P}^{1}\right)^{r}$ which maps $\left(0, z_{1}, \ldots, z_{r}, 1, \infty\right)$ to $\left(z_{1}, \ldots, z_{r}\right)$ sends $\Phi_{r}$ to the standard simplex $\Delta_{r}=\left\{0<t_{1}<\ldots<t_{r}<1\right\}$ and maps $B_{r}$ to the algebraic boundary of $\Delta_{r}$. A first sequence of blow-ups along the subvarieties $\left\{0=t_{1}=\ldots t_{i}\right\}$ corresponds to the change of variables from the simplicial to the cubical coordinates (6). In order to recover $B_{r}$, the blowup along the proper transform of the subvarieties $\left\{t_{i}=t_{i+1}=\ldots=t_{j}\right\}$ and $\left\{t_{i}=t_{i+1}=\ldots=t_{r}=1\right\}$ still has to be performed. The expression of these subvarieties in cubical coordinates is given by $\left\{x_{i}=x_{i+1}=\ldots=x_{j}=1\right\}$. The fact that it seems that we are blowing up less strata in order to recover $\overline{\mathcal{M}_{0, r+3}}$ from $\left(\mathbb{P}^{1}\right)^{r}$ using $\tilde{\delta_{r}}$ (25) comes from the fact that we are only looking at $\overline{\mathcal{M}_{0, r+3}} \backslash A_{r}$.

From the previous lemma we deduce

Corollary 4.26. (1) Let $\mathbf{a}=\left(a_{1}, \ldots, a_{b}\right)$ be a b-tuple of integers with $a_{1} \geqslant 2$ and $a_{1}+\cdots+a_{b}=n+m$. Using the previous convention, we have the following equality of framed mixed Tate motives

$$
\zeta^{f r \cdot \mathcal{M}}(\mathbf{a}, \mathrm{id})=\left[\mathrm{H}^{n+m}\left(\overline{\mathcal{M}_{0, n+m+3}} \backslash A_{0} ; B_{n+m}^{A_{0}}\right) ;\left[\omega_{\mathbf{a}}\right],\left[\Phi_{n+m}\right]\right] .
$$

(2) Let $\mathbf{k}$ and $\mathbf{l}$ be as in proposition 4.24, then there exist two distinguished flags $\left(\mathcal{F}, i_{1}, \ldots, i_{p}\right)$ and $\left(\mathcal{F}^{\prime}, j_{1}, \ldots, j_{q}\right)$ such that the length of $\mathcal{F}$ (resp. $\left.\mathcal{F}^{\prime}\right)$ is $i_{p}$ (resp. $j_{q}$ ) with $i_{1}, j_{1} \geqslant 2$ and the sets $I_{i_{p}}$ and $I_{j_{q}}^{\prime}$ form a partition of $\llbracket 1, n \rrbracket$. The following equality of framed mixed Tate motives holds

$$
\begin{aligned}
\zeta^{f r \cdot \mathcal{M}}\left(\mathcal{F}, i_{1}, \ldots i_{p} \mid \mathcal{F}^{\prime}, j_{1}, \ldots j_{q}\right)= & \\
& {\left[\mathrm{H}^{n+m}\left(\overline{\mathcal{M}_{0, n+m+3}} \backslash A_{0}, B_{n+m}^{A_{0}}\right) ;\left[\omega_{\mathbf{k}} \wedge \omega_{\mathbf{l}}\right],\left[\Phi_{n+m}\right]\right] . }
\end{aligned}
$$

As a consequence, for all $\sigma \in \mathrm{st}(\mathbf{k}, \mathbf{l})$, the framed mixed Tate motives

$$
\left[\mathrm{H}^{n+m}\left(\overline{\mathcal{M}_{0, n+m+3}} \backslash A_{0}, B_{n+m}^{A_{0}}\right) ;\left[\omega_{\sigma}\right],\left[\Phi_{n+m}\right]\right]
$$

are equal to the frame mixed motives $\zeta^{\text {fr.M }}(\sigma)$.

Proof. In 1. and 2., the map on the underlying vector space is given by $\alpha_{n+m}^{*}$ (cf. (25)). As $\widehat{C}_{n+m}$ is map to $\Phi_{n+m}$, knowing the behaviour of $\alpha_{n+m}^{*}$ with respect to the form $\omega_{\mathbf{a}}$ and $\omega_{\mathbf{k}} \wedge \omega_{\mathbf{l}}$ is enough to deduce that $\alpha_{n+m}^{*}$ respects the frames.

(1) As the the map $\alpha_{n+m}^{*}$ has no effect on the $u_{i}$ coordinates on $\overline{\mathcal{M}_{0, n+m+3}} \backslash A_{0}$, we have $\alpha_{n+m}^{*}\left(\omega_{\mathbf{a}}\right)=\omega_{\mathbf{a}}^{\text {id }}$, and thus the equality of framed mixed Tate motives.

(2) Writing down in cubical coordinates the expression

$$
\omega_{\mathbf{k}}=f_{\mathbf{k}}\left(u_{1}, \ldots, u_{n}\right) d^{n} u \quad \text { and } \quad \omega_{\mathbf{l}}=f_{\mathbf{k}}\left(u_{n+1}, \ldots, u_{n+m}\right) d^{m} u
$$

leads to the definition of two distinguished flags

$$
\left(\mathcal{F}, i_{1}, \ldots, i_{p}\right) \text { and }\left(\mathcal{F}^{\prime}, j_{1}, \ldots, j_{q}\right),
$$

as in remark 4.15 with $s=\mathrm{id}$. The fact that $\alpha_{n+m}^{*}$ respects the frames come from the equality

$$
\omega_{i_{1}, \ldots, i_{p}}^{\mathcal{F}^{\prime}} \wedge \omega_{j_{1}, \ldots, j_{q}}^{\mathcal{F}^{\prime}}=\alpha_{n+m}^{*}\left(\omega_{\mathbf{k}} \wedge \omega_{\mathbf{l}}\right) .
$$

The only thing that remains to be checked to complete the proof of proposition 4.24 is, using the notation of the previous lemma, that

$$
\zeta^{f r \cdot \mathcal{M}}\left(\mathcal{F}, i_{1}, \ldots i_{p} \mid \mathcal{F}^{\prime}, j_{1}, \ldots j_{q}\right)=\sum_{\sigma \in \operatorname{st}(\mathbf{k}, \mathbf{l})} \zeta^{f r \cdot \mathcal{M}}(\sigma, \text { id }) .
$$


Using the computation of section 1.3, in particular the proposition 1.5, we have that for each $\sigma \in \operatorname{st}(\mathbf{k}, \mathbf{l})$ there exists a permutation $s_{\sigma}$ such that

$$
\left[\omega_{i_{1}, \ldots, i_{p}}^{\mathcal{F}^{\prime}} \wedge \omega_{j_{1}, \ldots, j_{q}}^{\mathcal{F}^{\prime}}\right]=\sum_{\sigma \in \operatorname{st}(\mathbf{k}, \mathbf{l})}\left[\omega_{\sigma, s_{\sigma}}\right]
$$

As the divisor $A_{\mathcal{F}^{\prime}, j_{1}, \cdots, j_{q}}^{\mathcal{F}, i_{1}, \ldots, i_{p}}$ of $\omega_{i_{1}, \ldots, i_{p}}^{\mathcal{F}^{\prime}} \wedge \omega_{j_{1}, \ldots, j_{q}}^{\mathcal{F}^{\prime}}$ and the divisors $A_{\sigma, s_{\sigma}}$ are in $\widehat{A}_{n+m}$, lemma 3.6 and an analogue of lemma 3.3 show that

$$
\zeta^{f r \cdot \mathcal{M}}\left(\mathcal{F}, i_{1}, \ldots i_{p} \mid \mathcal{F}^{\prime}, j_{1}, \ldots j_{q}\right)=\sum_{\sigma \in \operatorname{st}(\mathbf{k}, \mathbf{l})} \zeta^{f r \cdot \mathcal{M}}\left(\sigma, s_{\sigma}\right) .
$$

Permuting the variables gives a well defined morphism $X_{n+m} \rightarrow X_{n+m}$ that preserves $\widehat{C}_{n+m}$ and its algebraic boundary $\widehat{B}_{n+m}$. It leads, on each term of the RHS of (27), to an equality

$$
\zeta^{f r \cdot \mathcal{M}}\left(\sigma, s_{\sigma}\right)=\zeta^{f r \cdot \mathcal{M}}(\sigma, \mathrm{id}),
$$

and hence to

$$
\zeta^{f r \cdot \mathcal{M}}\left(\mathcal{F}, i_{1}, \ldots i_{p} \mid \mathcal{F}^{\prime}, j_{1}, \ldots j_{q}\right)=\sum_{\sigma \in \operatorname{st}(\mathbf{k}, \mathbf{l})} \zeta^{f r \cdot \mathcal{M}}(\sigma, \text { id }) .
$$

and Proposition 4.24.

\section{REFERENCES}

[BGSV90] A. A. Beйlinson, A. B. Goncharov, V. V. Schechtman, and A. N. Varchenko, Aomoto dilogarithms, mixed Hodge structures and motivic cohomology of pairs of triangles on the plane, The Grothendieck Festschrift, Vol. I, Progr. Math., vol. 86, Birkhäuser Boston, Boston, MA, 1990, pp. 135-172.

[Bro06] Francis Brown, Multiple zeta values and periods of moduli spaces $\overline{\mathfrak{M}}_{0, n}(\mathbb{R})$., Ph.D. thesis, Université de Bordeaux, arXiv:mmath/0606419, 2006.

[GM04] A. B. Goncharov and Yu. I. Manin, Multiple $\zeta$-motives and moduli spaces $\overline{\mathcal{M}_{0, n}}$, Compos. Math. 140 (2004), no. 1, 1-14.

[Gon99] A. B. Goncharov, Volumes of hyperbolic manifolds and mixed Tate motives, J. Amer. Math. Soc. 12 (1999), no. 2, 569-618.

[Gon01] , Multiple polylogarithms and mixed tate motives, math.AG/0103059, May 2001.

[Gon02] _ Periods and mixed motives, www.arxiv.org/abs/math.AG/0202154, May 2002.

[Gon05] Galois symmetries of fundamental groupoids and noncommutative geometry, Duke Math. J. 128 (2005), no. 2, 209-284.

[Hu03] Yi Hu, A compactification of open varieties, Trans. Amer. Math. Soc. 355 (2003), no. 12, 4737-4753 (electronic).

[Uly02] Alexander P. Ulyanov, Polydiagonal compactification of configuration spaces, J. Algebraic Geom. 11 (2002), no. 1, 129-159.

[Voe00] V. Voevodsky, Triangulated category of motives over a field, Cycles, transfers, and motivic homology theories, Annals of Math. Studies, vol. 143, Princeton University Press., 2000.

Institut de Mathématiques de Jussieu (IMJ), Université Paris Diderot - Paris 7 , 175 rue du Chevaleret, 75013 Paris 